\title{
A Double-Strand Elastic Rod Theory
}

\author{
MAHER MOAKHER \& JOHN H. MADDOCKS
}

Communicated by S.S. ANTMAN

\begin{abstract}
Motivated by applications in the modeling of deformations of the DNA double helix, we construct a continuum mechanics model of two elastically interacting elastic strands. The two strands are described in terms of averaged, or macroscopic, variables plus an additional small, internal or microscopic, perturbation. We call this composite structure a birod. The balance laws for the macroscopic configuration variables of the birod can be cast in the form of a classic Cosserat rod model with coupling to the internal balance laws through the constitutive relations. The internal balance laws for the microstructure variables also take a mathematical form analogous to that for a Cosserat rod, but with coupling to the macroscopic system through terms corresponding to distributed force and couple loads.
\end{abstract}

\section{Introduction}

The theory of elastic rods has a long history. There now exists a hierarchy of detailed theories that both justify and generalize the original (most simplistic and yet fundamental) model of Euler and the Bernoullis to encompass such effects as shear, extension, non-uniformity, intrinsic curvature, and nonlinear constitutive relations, in both the static and dynamic settings. A comprehensive survey of both the history and contemporary state of the field can be found in ANTMAN [2].

An inspection of the literature makes evident the unsurprising fact that the primary motivation for the development of elastic rod theories has been the most obvious application, namely to construct a simplified model whose governing equations can predict good approximations for the solutions of the full three-dimensional field equations of macroscopic elasticity when the body at hand is appropriately loaded and is long and slender, e.g., wires, ropes, cables or beams. Nevertheless, over the last several decades, elastic rod models have been increasingly applied to the microscopic problem of describing and understanding the deformations and 
supercoiling of fragments of DNA molecules at various length scales. Mirroring the development of the original rod theories, the continuum mechanics models that have been exploited within the DNA context have been of increasing sophistication, and have successfully yielded ever more detailed information and insights, (see, e.g., $[5,6,16,17,24,25,28])$.

At this time, there is a fundamental difficulty limiting the range of applicability of continuum rod models to DNA. While understanding the folding and deformations of the DNA double helix as a single structure is undeniably important within the biological context, the full biological function of DNA is inextricably linked to the fact that it is a double helix, and the mechanisms for strand separation (also called melting or unzipping) need to be better understood. Consequently, in the past two decades, several works have been directed toward modeling the opening of the DNA double helix. A survey of the literature reveals that there are two major approaches to the strand separation problem, both based on discrete statistical mechanics. BENHAM [7] models the free energy required for the separation of a given number of contiguous base pairs as the sum of the energy needed to nucleate opening plus the energy needed to open a specified number of contiguous base pairs with a specific sequence. The necessary energy parameters are determined from thermodynamical considerations and experimental results. Comparison with experiment has shown that the numerical simulations based on this model are a valuable tool in predicting sequence-dependent sites along the DNA molecule that are particularly susceptible to strand separation [8].

In the second approach, BARBI et al. [4] use a geometric and dynamic model, which they call the twist opening model, to study structural transitions of the DNA double helix whose axis is constrained to be a straight line. In their model, each base pair is represented by a simplified system with only two degrees of freedom, the radius and an orientation angle. The hydrogen bonds linking the two bases in a base pair are modeled by a Morse potential. Their description is a generalization that combines the planar model of PEYRARD \& BISHOP [20] describing transverse DNA dynamics and the torsional model of YOMOSA [35]. In the small-amplitude approximation, the continuum limit of the difference equations of this model yields differential equations that admit soliton solutions. There are also more elaborate models that are extensions of that of Peyrard-Bishop, but we defer to the review article [14] and YAKUSHEVICH [33, 34] for further details. See also PROHOFSKY [21] who uses a model of chemical bond disruption to model DNA double helix melting.

It seems that there is a reasonable consensus within the field that it would be desirable to strengthen the connections between models focused solely on basepair opening, and models focused solely on deformations of the actual double-helix, with the objective of understanding how the overall deformations of DNA including twist and bend waves and focusing effects interact, initiate and inhibit localized melting of the double helix. It is currently impossible to decide whether or not such melting phenomena can usefully be modeled by a continuum rod theory, for the simple reason that, to the best of our knowledge, no appropriate double-stranded continuum rod theory exists. Accordingly, the objective of this article is to develop just such a theory. While our motivation is firmly rooted in the DNA application, in this article we make only the first step of constructing the field equations for a 
double-strand continuum theory, and leave an investigation of the level of utility of the model within the DNA (or any other) context to subsequent work. Consequently there is no mention of DNA beyond the Introduction.

It should also be remarked that several authors have developed multi-strand rod models of a different type, motivated both by DNA supercoiling and other physical systems, such as spun ropes (see, e.g., $[10,12,13,27]$ ). In DNA supercoiling a plectoneme is formed when distinct parts of the DNA double helix deform in space and touch each other, typically winding around each other to form a two-stranded helical structure. However in that context, each of the two strands is itself a double helix. The macroscopic analogy is two pieces of wire winding around each other, but free to slide. This is a contact problem, with the feature that slithering can occur, so that a specific point on one strand can change the point or points on the other strand with which it interacts. The theory developed here is not appropriate for such systems. Rather a macroscopic analogue of our model of the DNA double helix would be two wires that are brought close to each other through bending and twisting, and which are glued to each other with an elastic cement. Thus each point on one strand (i.e., a specific base) has a distinguished partner on the other strand. Our focus is the construction of a continuum model that allows an analysis of the interaction of the overall macroscopic deformation of the structure and the internal, or microscopic, strains and stresses, with the objective of understanding when internal stresses could be focused in such a way that the glue fails, or the DNA double helix melts.

In Section 2 we establish the kinematics of double-stranded systems. In particular we define the strains of an averaged structure that we call the macroscopic variables, and the strains of an internal microstructure. We call the composite object described by the macroscopic and microscopic strains a birod. The development of the kinematics is not entirely straightforward because it entails taking appropriate averages of rotations. It does lead naturally to a theory in which derivatives of strain-like variables enter the kinematics.

Our development could be described as a mixture theory (see, e.g., [22]), appropriate for rods. The only prior work in this direction of which we are aware is the mixture theory for stresses of an arbitrary number of rods described in [23]. But that work differs from ours in that it is assumed that all rods are coincident in the deformed configuration, and only the differences in the stresses are analyzed.

In Section 3 we derive the appropriate balance laws. Essentially by construction, the field equations governing the macroscopic composite variables for the birod take precisely the form of the dynamics of a Cosserat rod. Moreover (in a result unanticipated by us) the field equations for the internal microscopic variables also take the form of Cosserat rod dynamics, but with the same macroscopic center line and with terms which formally correspond to distributed loading by external forces and moments (and which are in fact related to coupling with the macroscopic system). In Section 4 we start our consideration of constitutive relations for birods. In Section 5 we introduce an appropriate action principle for hyper-elastic birods, and use it to compute specific energies that are invariant under Euclidean motions. We clarify the circumstances in which the action principle implies the previously obtained balance laws. The internal structure variables involve derivatives that are 
reminiscent of strain-gradient theories, but in our context it is the relative displacements between strands which play the role of strains, and derivatives of these relative displacements play the role of strain gradients. The action principle also provides the appropriate natural boundary conditions for our birod. In Section 6 we discuss in more detail the specific case of an energy that is a simple quadratic function of a natural set of variables. In Section 7 we make our theory concrete in some very elementary, illustrative boundary-value problems. Our current conclusions and possible directions for continuation are discussed in Section 8.

\section{Kinematics}

\subsection{Preliminaries}

Let $\left\{O ; \boldsymbol{e}_{1}, \boldsymbol{e}_{2}, \boldsymbol{e}_{3}\right\}$ be a fixed frame, with origin $O$ and right-handed orthonormal basis $\left\{\boldsymbol{e}_{1}, \boldsymbol{e}_{2}, \boldsymbol{e}_{3}\right\}$, of Euclidean space $\mathscr{E}^{3}$. For any two vectors $\boldsymbol{a}, \boldsymbol{b} \in \mathscr{E}^{3}$, the scalar and vector products are denoted $\boldsymbol{a} \cdot \boldsymbol{b}$ and $\boldsymbol{a} \times \boldsymbol{b}$. The Euclidean norm of a vector $\boldsymbol{a}$ is denoted $\|\boldsymbol{a}\|=(\boldsymbol{a} \cdot \boldsymbol{a})^{1 / 2}$. The tensor product $\boldsymbol{a} \otimes \boldsymbol{b}$ of the vectors $\boldsymbol{a}$ and $\boldsymbol{b}$ is the second-order tensor defined by $(\boldsymbol{a} \otimes \boldsymbol{b}) \boldsymbol{c}=(\boldsymbol{c} \cdot \boldsymbol{b}) \boldsymbol{a}$ for all vectors $c \in \mathscr{E}^{3}$. For the vector $\boldsymbol{a}$, we denote by $\boldsymbol{a}^{\times}$the skew-symmetric tensor such that $\left(\boldsymbol{a}^{\times}\right) \boldsymbol{b}=\boldsymbol{a} \times \boldsymbol{b}$ for all vectors $\boldsymbol{b} \in \mathscr{E}^{3}$. Throughout the article, the summation convention for repeated Latin indices is employed with range 1 to 3 .

\subsection{Geometric description of birods}

We consider a birod $\mathscr{R}$ composed of two thin elastic rods $\mathscr{R}^{+}$and $\mathscr{R}^{-}$that are bound together elastically. The two rods will be referred to as the strands of the birod. Each strand is considered to be a special Cosserat rod [2] whose configuration is described by specifying, for each $s \in[0, L]$ and for each time $t$, a position vector (from the point $O$ ) and a right-handed triad of orthonormal directors:

$$
\begin{array}{ll}
\boldsymbol{r}^{+}(s, t), & \text { position vector for } \mathscr{R}^{+}, \\
\left\{\boldsymbol{d}_{1}^{+}(s, t), \boldsymbol{d}_{2}^{+}(s, t), \boldsymbol{d}_{3}^{+}(s, t)\right\}, & \text { directors for } \mathscr{R}^{+}, \\
\boldsymbol{r}^{-}(s, t), & \text { position vector for } \mathscr{R}^{-}, \\
\left\{\boldsymbol{d}_{1}^{-}(s, t), \boldsymbol{d}_{2}^{-}(s, t), \boldsymbol{d}_{3}^{-}(s, t)\right\}, & \text { directors for } \mathscr{R}^{-} .
\end{array}
$$

We will repeatedly make statements that apply equally to the + and - strands. Therefore, we adopt once and for all notation such as $\boldsymbol{r}^{ \pm}(s, t)$ to refer simultaneously to either strand with the convention that the upper sign of any quantity is associated with the upper sign of any other quantity.

The curves $\mathscr{C}^{ \pm}(t) \equiv\left\{\boldsymbol{r}^{ \pm}(s, t), s \in[0, L]\right\}$ represent the lines of centroids, in the current configuration, of $\mathscr{R}^{ \pm}$. We choose to parametrize these curves by a common parameter $s$ which need not be an arc length in either curve. The triads 
$\left\{\boldsymbol{d}_{1}^{ \pm}(s, t), \boldsymbol{d}_{2}^{ \pm}(s, t), \boldsymbol{d}_{3}^{ \pm}(s, t)\right\}$ give the orientation of the material cross section at $s$ of $\mathscr{R}^{ \pm}$. We take $\boldsymbol{R}^{ \pm}(s, t)$ to denote the proper orthogonal tensors

$$
\boldsymbol{R}^{ \pm}(s, t)=\boldsymbol{d}_{i}^{ \pm}(s, t) \otimes \boldsymbol{e}_{i}
$$

Throughout this article, quantities that are associated with the reference configuration are denoted with the same symbol as those associated with the actual configuration, but with a superposed circumflex, e.g., $\boldsymbol{r}$ and $\widehat{\boldsymbol{r}}$, etc. Thus, in the reference configuration the position vector and the triad of directors of $\mathscr{R}^{ \pm}$at $s$ are denoted by $\widehat{\boldsymbol{r}}^{ \pm}(s)$ and $\left\{\widehat{\boldsymbol{d}}_{1}^{ \pm}(s), \widehat{\boldsymbol{d}}_{2}^{ \pm}(s), \widehat{\boldsymbol{d}}_{3}^{ \pm}(s)\right\}$. We assume that $\widehat{\boldsymbol{d}}_{3}^{ \pm}(s)$ coincides with the unit tangent $\widehat{\boldsymbol{t}}^{ \pm}(s)$ to the curve $\widehat{\mathscr{C}}^{ \pm} \equiv\left\{\widehat{\boldsymbol{r}}^{ \pm}(s), s \in[0, L]\right\}$.

During the detailed development in this and the following section we shall assume that at each $s$ the material cross sections of both strands have mass densities given by

$$
\rho^{ \pm}(s)=\frac{1}{2} \lambda^{ \pm}(s) \rho(s)
$$

where the positive functions $\rho(s)$ and $\lambda^{ \pm}(s)$ are smooth, and that for all $s$

$$
0 \leqq \lambda^{ \pm}(s) \leqq 2, \quad \lambda^{+}(s)+\lambda^{-}(s)=2 .
$$

For convenience, we set $\lambda(s)=2-\lambda^{+}(s)=\lambda^{-}(s)$. The equal mass density theory can be recovered by taking $\lambda(s)=\lambda^{+}(s)=\lambda^{-}(s) \equiv 1$.

In this study, we switch attention from the two individual strands to a composite structure, which we call a birod, made up of an elastic rod describing the average or macroscopic deformations of the two strands, plus an internal, or microscopic, set of variables that capture the differences in deformations of the two strands. This approach falls under the general umbrella of continuum models endowed with internal microstructures [9] or mixture theories [22]. One justification for the introduction of a birod is the implicit assumption that the microscopic strains are small, in which case the macroscopic deformation of the birod already provides a good approximation to both individual strands, and the microscopic variables provide a correction.

The gross, macroscopic deformation of the birod is given by

$$
\begin{array}{ll}
\boldsymbol{r}(s, t), & \text { position vector for the birod, } \\
\left\{\boldsymbol{d}_{1}(s, t), \boldsymbol{d}_{2}(s, t), \boldsymbol{d}_{3}(s, t)\right\}, & \text { directors for the birod, }
\end{array}
$$

where the position vector $\boldsymbol{r}(s, t)$ is the weighted average of the position vectors of the two strands, i.e.,

$$
\boldsymbol{r}(s, t)=\frac{1}{2}\left[\lambda^{+}(s) \boldsymbol{r}^{+}(s, t)+\lambda^{-}(s) \boldsymbol{r}^{-}(s, t)\right],
$$

and the triad $\left\{\boldsymbol{d}_{1}(s, t), \boldsymbol{d}_{2}(s, t), \boldsymbol{d}_{3}(s, t)\right\}$ is given by

$$
\boldsymbol{d}_{i}(s, t)=\boldsymbol{R}(s, t) \boldsymbol{e}_{i}, \quad i=1,2,3,
$$


where the proper orthogonal tensor $\boldsymbol{R}(s, t)$ is the particular average of the proper orthogonal tensors $\boldsymbol{R}^{ \pm}(s, t)$ explicitly given by any of the four equivalent expressions [19]

$$
\begin{aligned}
\boldsymbol{R}(s, t) & =\boldsymbol{R}^{-}(s, t)\left[\boldsymbol{R}^{-}(s, t)^{T} \boldsymbol{R}^{+}(s, t)\right]^{1 / 2} \\
& =\boldsymbol{R}^{+}(s, t)\left[\boldsymbol{R}^{+}(s, t)^{T} \boldsymbol{R}^{-}(s, t)\right]^{1 / 2} \\
& =\left[\boldsymbol{R}^{+}(s, t) \boldsymbol{R}^{-}(s, t)^{T}\right]^{1 / 2} \boldsymbol{R}^{-}(s, t) \\
& =\left[\boldsymbol{R}^{-}(s, t) \boldsymbol{R}^{+}(s, t)^{T}\right]^{1 / 2} \boldsymbol{R}^{+}(s, t) .
\end{aligned}
$$

Here, for any proper orthogonal tensor $Q, Q^{1 / 2}$ denotes the proper orthogonal tensor solution of $\boldsymbol{X}^{2}=\boldsymbol{Q}$ for which the eigenvalues have the largest positive real part. Such a square root is well defined and unique unless $\boldsymbol{Q}$ is an involution, i.e., a rotation through the angle $\pi$. The proper orthogonal tensor $\boldsymbol{R}(s, t)$ defined in (2.8) is such that $\mathrm{d}\left(\boldsymbol{R}, \boldsymbol{R}^{+}\right)=\mathrm{d}\left(\boldsymbol{R}, \boldsymbol{R}^{-}\right)$, where $\mathrm{d}(\cdot, \cdot)$ is any properly invariant distance function in the group of rotations. The rotation tensor $\boldsymbol{R}(s, t)$ has another characterization: it is the polar factor in the polar decomposition of the tensor $\left[\boldsymbol{R}^{+}(s, t)+\boldsymbol{R}^{-}(s, t)\right]$, cf. [19].

The microstructure of the birod is described by a microdisplacement and a microrotation. The translational microdisplacement is

$$
\boldsymbol{w}(s, t)=\frac{1}{2}\left[\boldsymbol{r}^{+}(s, t)-\boldsymbol{r}^{-}(s, t)\right],
$$

whereas the microrotation can be described either through

$$
\boldsymbol{P}(s, t)=\left[\boldsymbol{R}^{+}(s, t) \boldsymbol{R}^{-}(s, t)^{T}\right]^{1 / 2},
$$

or

$$
\boldsymbol{O}(s, t)=\left[\boldsymbol{R}^{-}(s, t)^{T} \boldsymbol{R}^{+}(s, t)\right]^{1 / 2} .
$$

If the birod is described by its center line $\boldsymbol{r}(s, t)$, the proper orthogonal tensor $\boldsymbol{R}(s, t)$, the microdisplacement $\boldsymbol{w}(s, t)$ and either of the microrotations $\boldsymbol{P}(s, t)$ or $\boldsymbol{O}(s, t)$, then the configurations of the two strands can be recovered from the relations

$$
\begin{gathered}
\boldsymbol{r}^{ \pm}(s, t)=\boldsymbol{r}(s, t) \pm \lambda^{\mp}(s) \boldsymbol{w}(s, t), \\
\boldsymbol{R}^{+}(s, t)=\boldsymbol{P}(s, t) \boldsymbol{R}(s, t)=\boldsymbol{R}(s, t) \boldsymbol{O}(s, t), \\
\boldsymbol{R}^{-}(s, t)=\boldsymbol{P}(s, t)^{T} \boldsymbol{R}(s, t)=\boldsymbol{R}(s, t) \boldsymbol{O}(s, t)^{T} .
\end{gathered}
$$

Because of the relations $\left(2.13^{ \pm}\right)$, and in analogy with the polar decomposition of the deformation gradient in continuum mechanics, we will call $\boldsymbol{O}(s, t)$ the right microrotation and $\boldsymbol{P}(s, t)$ the left microrotation. We note that these two microrotations are related through

$$
\boldsymbol{O}(s, t)=\boldsymbol{R}(s, t)^{T} \boldsymbol{P}(s, t) \boldsymbol{R}(s, t) .
$$


We have consistently adopted the notation \pm to refer to objects associated with the plus and minus strands. To continue this convention it is convenient to introduce the notation

$$
\boldsymbol{P}^{+}:=\boldsymbol{P} \text { and } \boldsymbol{P}^{-}:=\boldsymbol{P}^{T}=\boldsymbol{P}^{-1},
$$

so that from $\left(2.13^{ \pm}\right)$we have

$$
\boldsymbol{R}^{ \pm}(s, t)=\boldsymbol{P}^{ \pm}(s, t) \boldsymbol{R}(s, t) .
$$

From $\left(2.3^{ \pm}\right),(2.7)$ and $\left(2.15^{ \pm}\right)$one can see that

$$
\boldsymbol{P}^{ \pm}=\boldsymbol{d}_{i}^{ \pm} \otimes \boldsymbol{d}_{i}
$$

and hence the rotation tensor $\boldsymbol{P}^{ \pm}$maps the directors $\left\{\boldsymbol{d}_{i}\right\}$ associated with the center line $\boldsymbol{r}$ onto the directors $\left\{\boldsymbol{d}_{i}^{ \pm}\right\}$associated with the center line $\boldsymbol{r}^{ \pm}$. Figure 1 illustrates the relations between the different frames of directors. The rotation tensor $\boldsymbol{O}$ transforms coordinates of vectors with respect to the three frames associated with $\boldsymbol{R}$, and $\boldsymbol{R}^{ \pm}$. We shall not consider $\boldsymbol{O}$ any further, but instead work exclusively with $\boldsymbol{P}$.

We shall assume that $s$ is an arc-length parameter for the curve $\widehat{\mathscr{C}}$, i.e., the center line of the birod in its reference configuration. Let $s^{ \pm}$be arc-length parameters for the reference curves $\widehat{\mathscr{C}}^{ \pm}$with the same orientation as $s$. (We note that in the standard forms of DNA the two strands are most naturally considered to be anti-parallel, but that modeling decision can be incorporated later.) Because $s$ is

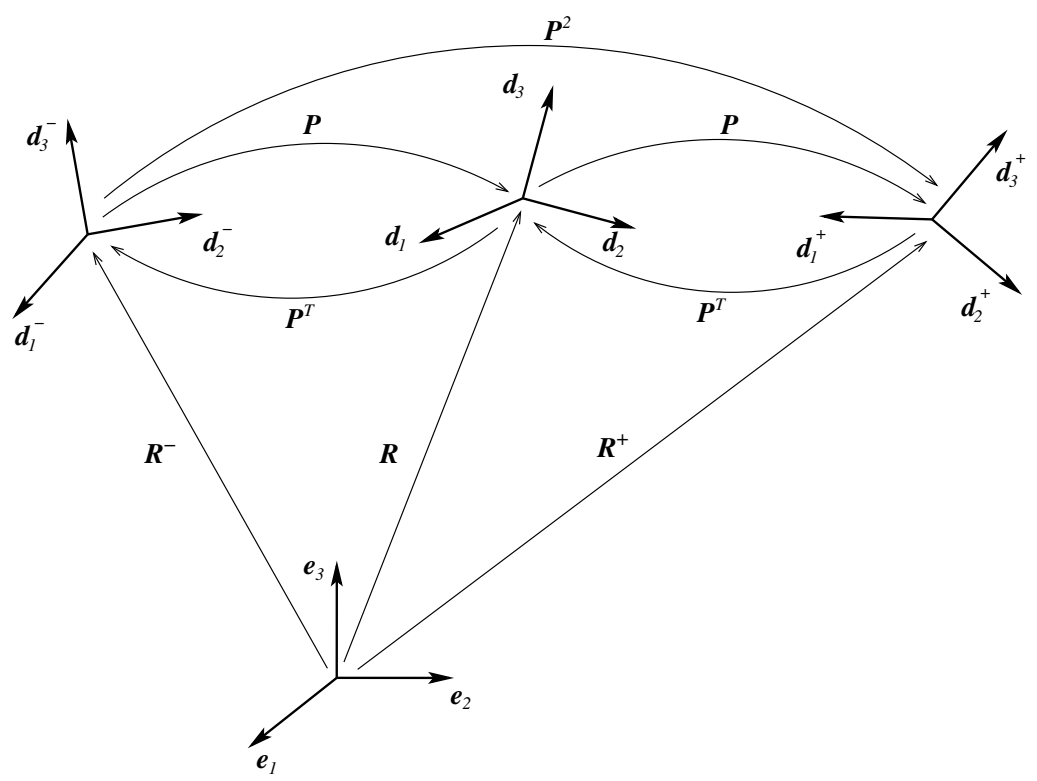

Figure 1. Relations between the rotation tensors $\boldsymbol{R}, \boldsymbol{R}^{ \pm}$, and $\boldsymbol{P}$, the director frames $\left\{\boldsymbol{d}_{i}\right\},\left\{\boldsymbol{d}_{i}^{ \pm}\right\}$, and the fixed frame $\left\{\boldsymbol{e}_{i}\right\}$. 
an arc-length parameter for $\widehat{\mathscr{C}}$ we have, from $\left(2.12^{ \pm}\right)$evaluated on the reference configuration,

$$
\widehat{\boldsymbol{r}}_{s}^{ \pm}(s) \cdot \widehat{\boldsymbol{r}}_{s}^{ \pm}(s)=1+\left(\lambda^{\mp}(s) \widehat{\boldsymbol{w}}(s)\right)_{s} \cdot\left[\left(\lambda^{\mp}(s) \widehat{\boldsymbol{w}}(s)\right)_{s} \pm 2 \widehat{\boldsymbol{r}}_{s}(s)\right] .
$$

We will assume that

$$
-1<\left(\lambda^{\mp}(s) \widehat{\boldsymbol{w}}(s)\right)_{s} \cdot\left[\left(\lambda^{\mp}(s) \widehat{\boldsymbol{w}}(s)\right)_{s} \pm 2 \widehat{\boldsymbol{r}}_{s}(s)\right]<\infty,
$$

so that the positive functions $g^{ \pm}(s)$ defined by

$$
g^{ \pm}(s)^{2}=1+\left(\lambda^{\mp}(s) \widehat{\boldsymbol{w}}(s)\right)_{s} \cdot\left[\left(\lambda^{\mp}(s) \widehat{\boldsymbol{w}}(s)\right)_{s} \pm 2 \widehat{\boldsymbol{r}}_{s}(s)\right]
$$

are smooth. Hence, the functions $s^{ \pm}(s)$, relating the three arc-lengths, are oneto-one with

$$
\frac{d s^{ \pm}}{d s}=g^{ \pm}(s) .
$$

The functions $g^{ \pm}(s)$ will be called the relative stretches of the curves $\widehat{\mathscr{C}}^{ \pm}$with respect to the curve $\widehat{\mathscr{C}}$.

\subsection{Strains and velocities}

The kinematics associated with the center lines $\boldsymbol{r}(s, t)$ and $\boldsymbol{r}^{ \pm}(s, t)$ are given by

$$
\begin{aligned}
\boldsymbol{v}(s, t) & :=\frac{\partial \boldsymbol{r}(s, t)}{\partial s}, \\
\boldsymbol{v}^{ \pm}(s, t) & :=\frac{\partial \boldsymbol{r}^{ \pm}(s, t)}{\partial s^{ \pm}}=\frac{1}{g^{ \pm}(s)} \frac{\partial \boldsymbol{r}^{ \pm}(s, t)}{\partial s} .
\end{aligned}
$$

Using $\left(2.12^{ \pm}\right)$and $\left(2.18^{ \pm}\right)$we can relate the strains $v^{ \pm}(s, t)$ to the macrostrain $\boldsymbol{v}(s, t)$ and the microdisplacement $\boldsymbol{w}(s, t)$, i.e.,

$$
\boldsymbol{v}^{ \pm}(s, t)=\frac{1}{g^{ \pm}(s)}\left[\boldsymbol{v}(s, t) \pm \lambda^{\mp}(s) \boldsymbol{w}_{s}(s, t) \pm \lambda_{s}^{\mp}(s) \boldsymbol{w}(s, t)\right] .
$$

Here and throughout the article, a subscript $s$ or $t$ denotes partial differentiation with respect to the indicated variable.

The linear velocities $\boldsymbol{\gamma}^{ \pm}(s, t) \equiv \boldsymbol{r}_{t}^{ \pm}(s, t)$ of the strands are related to the linear velocity $\boldsymbol{\gamma}(s, t) \equiv \boldsymbol{r}_{t}(s, t)$ of the birod and the time derivative of the microdisplacement $\boldsymbol{w}(s, t)$ through

$$
\boldsymbol{\gamma}^{ \pm}(s, t)=\boldsymbol{\gamma}(s, t) \pm \lambda^{\mp}(s) \boldsymbol{w}_{t}(s, t) .
$$

Let $\boldsymbol{U}(s, t)$ and $\boldsymbol{U}^{ \pm}(s, t)$ be the skew-symmetric tensors such that

$$
\begin{gathered}
\frac{\partial}{\partial s} \boldsymbol{R}(s, t)=\boldsymbol{U}(s, t) \boldsymbol{R}(s, t), \\
\frac{\partial}{\partial s^{ \pm}} \boldsymbol{R}^{ \pm}(s, t)=\boldsymbol{U}^{ \pm}(s, t) \boldsymbol{R}^{ \pm}(s, t) .
\end{gathered}
$$


With these skew-symmetric tensors we associate the corresponding Darboux vectors $\boldsymbol{u}(s, t)$ and $\boldsymbol{u}^{ \pm}(s, t)$, i.e., for any vector $\boldsymbol{a}$ we have

$$
\begin{aligned}
\boldsymbol{U}(s, t) \boldsymbol{a} & =\boldsymbol{u}(s, t) \times \boldsymbol{a}, \\
\boldsymbol{U}^{ \pm}(s, t) \boldsymbol{a} & =\boldsymbol{u}^{ \pm}(s, t) \times \boldsymbol{a} .
\end{aligned}
$$

Similarly, let $\boldsymbol{\Omega}(s, t)$ and $\boldsymbol{\Omega}^{ \pm}(s, t)$ be the skew-symmetric tensors such that

$$
\begin{gathered}
\frac{\partial}{\partial t} \boldsymbol{R}(s, t)=\boldsymbol{\Omega}(s, t) \boldsymbol{R}(s, t), \\
\frac{\partial}{\partial t} \boldsymbol{R}^{ \pm}(s, t)=\boldsymbol{\Omega}^{ \pm}(s, t) \boldsymbol{R}^{ \pm}(s, t),
\end{gathered}
$$

and let $\omega(s, t)$ and $\omega^{ \pm}(s, t)$, be the angular velocities such that

$$
\begin{aligned}
\boldsymbol{\Omega}(s, t) \boldsymbol{a} & =\boldsymbol{\omega}(s, t) \times \boldsymbol{a}, \\
\boldsymbol{\Omega}^{ \pm}(s, t) \boldsymbol{a} & =\boldsymbol{\omega}^{ \pm}(s, t) \times \boldsymbol{a} .
\end{aligned}
$$

We define $\boldsymbol{\Psi}^{ \pm}(s, t)$ and $\boldsymbol{\Phi}^{ \pm}(s, t)$ to be the spatial and time rates of rotation of $\boldsymbol{P}^{ \pm}(s, t)$,

$$
\begin{aligned}
\frac{\partial}{\partial s} \boldsymbol{P}^{ \pm}(s, t) & =\Psi^{ \pm}(s, t) \boldsymbol{P}^{ \pm}(s, t), \\
\frac{\partial}{\partial t} \boldsymbol{P}^{ \pm}(s, t) & =\boldsymbol{\Phi}^{ \pm}(s, t) \boldsymbol{P}^{ \pm}(s, t),
\end{aligned}
$$

and let $\boldsymbol{\psi}^{ \pm}(s, t)$ and $\boldsymbol{\phi}^{ \pm}(s, t)$ be the corresponding axial vectors, i.e., for any vector $\boldsymbol{a}$,

$$
\begin{aligned}
& \boldsymbol{\Psi}^{ \pm}(s, t) \boldsymbol{a}=\boldsymbol{\psi}^{ \pm}(s, t) \times \boldsymbol{a} \\
& \boldsymbol{\Phi}^{ \pm}(s, t) \boldsymbol{a}=\boldsymbol{\phi}^{ \pm}(s, t) \times \boldsymbol{a} .
\end{aligned}
$$

Note that we have the relations

$$
\begin{aligned}
& \boldsymbol{\Psi}^{ \pm}(s, t)=-\boldsymbol{P}^{\mp}(s, t)^{T} \boldsymbol{\Psi}^{\mp}(s, t) \boldsymbol{P}^{\mp}(s, t), \\
& \boldsymbol{\Phi}^{ \pm}(s, t)=-\boldsymbol{P}^{\mp}(s, t)^{T} \boldsymbol{\Phi}^{\mp}(s, t) \boldsymbol{P}^{\mp}(s, t),
\end{aligned}
$$

and

$$
\begin{aligned}
& \boldsymbol{\psi}^{ \pm}(s, t)=-\boldsymbol{P}^{\mp}(s, t)^{T} \boldsymbol{\psi}^{\mp}(s, t), \\
& \boldsymbol{\phi}^{ \pm}(s, t)=-\boldsymbol{P}^{\mp}(s, t)^{T} \boldsymbol{\phi}^{\mp}(s, t) .
\end{aligned}
$$

The skew-symmetric tensors $\boldsymbol{U}^{ \pm}(s, t)$ are related to the skew-symmetric tensor $\boldsymbol{U}(s, t)$ through

$$
\boldsymbol{U}^{ \pm}=\frac{1}{g^{ \pm}}\left[\boldsymbol{P}^{ \pm} \boldsymbol{U} \boldsymbol{P}^{ \pm T}+\boldsymbol{\Psi}^{ \pm}\right]=\frac{1}{g^{ \pm}} \boldsymbol{P}^{ \pm}\left[\boldsymbol{U}-\boldsymbol{\Psi}^{\mp}\right] \boldsymbol{P}^{ \pm T}
$$

Therefore, the strains associated with the directors of $\mathscr{R}^{ \pm}$are

$$
\boldsymbol{u}^{ \pm}=\frac{1}{g^{ \pm}}\left[\boldsymbol{P}^{ \pm} \boldsymbol{u}+\boldsymbol{\psi}^{ \pm}\right]=\frac{1}{g^{ \pm}} \boldsymbol{P}^{ \pm}\left[\boldsymbol{u}-\boldsymbol{\psi}^{\mp}\right] .
$$


Similarly, the skew-symmetric tensors $\boldsymbol{\Omega}^{ \pm}(s, t)$ are related to the skew-symmetric tensor $\boldsymbol{\Omega}(s, t)$ through

$$
\boldsymbol{\Omega}^{ \pm}=\boldsymbol{P}^{ \pm} \boldsymbol{\Omega} \boldsymbol{P}^{ \pm T}+\boldsymbol{\Phi}^{ \pm}=\boldsymbol{P}^{\mp}\left[\boldsymbol{\Omega}-\boldsymbol{\Phi}^{\mp}\right] \boldsymbol{P}^{ \pm T} .
$$

Therefore, the angular velocities associated with $\mathscr{R}^{ \pm}$are

$$
\omega^{ \pm}=\boldsymbol{P}^{ \pm} \boldsymbol{\omega}+\boldsymbol{\phi}^{ \pm}=\boldsymbol{P}^{ \pm}\left[\boldsymbol{\omega}-\boldsymbol{\phi}^{\mp}\right] .
$$

For any vector-valued function $\boldsymbol{b}(s, t)$, we shall adopt the notation

$$
\begin{gathered}
\boldsymbol{b}^{\nabla}=\boldsymbol{b}_{s}-\boldsymbol{u} \times \boldsymbol{b}=\boldsymbol{b}_{s}-\boldsymbol{R}_{s} \boldsymbol{R}^{T}, \\
\stackrel{\circ}{\boldsymbol{b}}=\boldsymbol{b}_{t}-\boldsymbol{\omega} \times \boldsymbol{b}=\boldsymbol{b}_{t}-\boldsymbol{R}_{t} \boldsymbol{R}^{T} .
\end{gathered}
$$

That is $\boldsymbol{b}^{\nabla}$ is the convected $s$-derivative of $\boldsymbol{b}$ relative to the moving frame $\left\{\boldsymbol{d}_{1}(s, t), \boldsymbol{d}_{2}(s, t), \boldsymbol{d}_{3}(s, t)\right\}$, while $\stackrel{\circ}{b}$ is the convected time derivative of $\boldsymbol{b}$ relative to this frame.

We now give relations between $\boldsymbol{\psi}^{ \pm}(s, t)$ and $\boldsymbol{\phi}^{ \pm}(s, t)$, and the spatial and time rates of change of the rotation $\boldsymbol{P}^{ \pm}(s, t)$. Let $\boldsymbol{k}(s, t)$ be the unit vector such that $\boldsymbol{P}(s, t) \boldsymbol{k}(s, t)=\boldsymbol{k}(s, t)$, and $\theta(s, t)$ be the angle of rotation of $\boldsymbol{P}(s, t)$, i.e., $\operatorname{tr} \boldsymbol{P}(s, t)=1+2 \cos \theta(s, t)$. Then referring to [1, p. 44], we have

$$
\begin{aligned}
& \boldsymbol{\psi}^{ \pm}= \pm \theta_{s} \boldsymbol{k} \pm \sin \theta \boldsymbol{k}_{s}+(1-\cos \theta) \boldsymbol{k} \times \boldsymbol{k}_{s}, \\
& \boldsymbol{\phi}^{ \pm}= \pm \theta_{t} \boldsymbol{k} \pm \sin \theta \boldsymbol{k}_{t}+(1-\cos \theta) \boldsymbol{k} \times \boldsymbol{k}_{t} .
\end{aligned}
$$

If we denote by $\boldsymbol{\theta}(s, t)$ the principal rotation vector of $\boldsymbol{P}$, i.e., $\boldsymbol{\theta}(s, t)=\theta(s, t)$ $\boldsymbol{k}(s, t)$, then, after some algebra, we obtain

$$
\boldsymbol{\psi}^{ \pm}=\left(\boldsymbol{I}-\boldsymbol{P}^{ \pm}\right) \boldsymbol{u} \pm \operatorname{sinc}(\theta / 2) \boldsymbol{P}^{ \pm 1 / 2} \boldsymbol{\theta}^{\nabla} \pm\left(\boldsymbol{k} \cdot \boldsymbol{\theta}^{\nabla}\right)(1-\operatorname{sinc}(\theta / 2)) \boldsymbol{k}
$$

and

$$
\boldsymbol{\phi}^{ \pm}=\left(\boldsymbol{I}-\boldsymbol{P}^{ \pm}\right) \boldsymbol{\omega} \pm \operatorname{sinc}(\theta / 2) \boldsymbol{P}^{ \pm 1 / 2} \stackrel{\circ}{\boldsymbol{\theta}} \pm(\boldsymbol{k} \cdot \stackrel{\circ}{\boldsymbol{\theta}})(1-\operatorname{sinc}(\theta / 2)) \boldsymbol{k}
$$

where $\operatorname{sinc}(\cdot)$ is the cardinal sine function defined on the real line by $\operatorname{sinc}(x)=$ $\sin (x) / x$, if $x \neq 0$ and $\operatorname{sinc}(0)=1$. The above formulæ are cumbersome. However, if we introduce the Gibbs rotation vector

$$
\boldsymbol{\eta}(s, t)=\tan (\theta(s, t) / 2) \boldsymbol{k}(s, t),
$$

then we have the more compact formulæ

$$
\boldsymbol{\psi}^{ \pm}=\left(\boldsymbol{I}-\boldsymbol{P}^{ \pm}\right) \boldsymbol{u} \pm \alpha \boldsymbol{A}^{ \pm} \boldsymbol{\eta}^{\nabla},
$$

and

$$
\boldsymbol{\phi}^{ \pm}=\left(\boldsymbol{I}-\boldsymbol{P}^{ \pm}\right) \boldsymbol{\omega} \pm \alpha \boldsymbol{A}^{ \pm}{ }_{\boldsymbol{\eta}}^{\circ},
$$

where

$$
\boldsymbol{A}^{ \pm}=\boldsymbol{I} \pm \eta^{\times},
$$


and

$$
\alpha=\frac{2}{1+\|\eta\|^{2}} .
$$

Substitution of $\left(2.45^{ \pm}\right)$into $\left(2.35^{ \pm}\right)$yields

$$
\boldsymbol{u}^{ \pm}=\frac{1}{g^{ \pm}}\left[\boldsymbol{u} \pm \alpha \boldsymbol{A}^{ \pm} \boldsymbol{\eta}^{\nabla}\right] \text {. }
$$

Similarly, substitution of $\left(2.46^{ \pm}\right)$into $\left(2.37^{ \pm}\right)$yields

$$
\omega^{ \pm}=\omega \pm \alpha A^{ \pm} \stackrel{\circ}{\eta}
$$

The relations $\left(2.49^{ \pm}\right)$and $\left(2.50^{ \pm}\right)$are geometrically exact. From the analytical point of view, the use of the Gibbs vector, also called Rodrigues parameters [32], seems, in our case, to be preferable to any other parameterizations of the rotation $\boldsymbol{P}=\boldsymbol{P}^{+}=\boldsymbol{P}^{-T}$. With other parameterizations the expressions relating the rate of change of $\boldsymbol{P}$ with the rate of change of the parameter can be quite complicated. For instance, the use of the principal rotation vector leads to $\left(2.42^{ \pm}\right)$and $\left(2.43^{ \pm}\right)$. The Gibbs vector provides a minimal parameterization of the rotation $\boldsymbol{P}$, that only becomes singular when $\boldsymbol{P}$ is a rotation through an angle $\pi$. And for the description of an a priori small microstructure variables this restriction seems unimportant. We note that the Gibbs vector is closely related to the singularity-free four-parameter quaternion representation of rotation. In fact, if $\left[q_{0}, \boldsymbol{q}\right]$ is the unit quaternion (also known as the Euler parameters) of the rotation $\boldsymbol{P}$, then the Gibbs vector $\boldsymbol{\eta}$ is simply the vector $\boldsymbol{q}$ rescaled by the scalar part $q_{0}$, i.e., $\boldsymbol{\eta}=\boldsymbol{q} / q_{0}$.

In summary, the relevant kinematic variables are the strains $v(s, t)$ and $\boldsymbol{v}^{ \pm}(s, t)$ associated with the center lines, the strains $\boldsymbol{u}(s, t)$ and $\boldsymbol{u}^{ \pm}(s, t)$ associated with the directors, the linear velocities $\boldsymbol{\gamma}(s, t)$ and $\boldsymbol{\gamma}^{ \pm}(s, t)$, the angular velocities $\boldsymbol{\omega}(s, t)$ and $\omega^{ \pm}(s, t)$, the vectors $\boldsymbol{w}(s, t), \boldsymbol{w}_{s}(s, t)$ and $\boldsymbol{w}_{t}(s, t)$ associated with the internal microtranslation, and the vectors $\eta(s, t), \eta_{s}(s, t)$ and $\eta_{t}(s, t)$ associated with the internal microrotation. Nevertheless, we find it more convenient, especially in Section 5, to work with the convected derivatives $\boldsymbol{w}^{\nabla}$ and $\stackrel{\circ}{\boldsymbol{w}}$ instead of $\boldsymbol{w}_{s}$ and $\boldsymbol{w}_{t}$, and with $\alpha \boldsymbol{\eta}^{\nabla}$ and $\alpha \stackrel{\circ}{\eta}$ instead of $\boldsymbol{\eta}_{s}$ and $\boldsymbol{\eta}_{t}$ where the function $\alpha$ was introduced in (2.48). At first sight the choice of the variable $\alpha \boldsymbol{\eta}^{\nabla}$ for representing $\boldsymbol{P}_{s}$ seems artificial in comparison to other possibilities. In particular, equations $\left(2.45^{ \pm}\right)$suggest that the variable $\boldsymbol{\varphi}^{+}=\alpha \boldsymbol{A}^{+} \boldsymbol{\eta}^{\nabla}$ seems appropriate for representing $\boldsymbol{P}_{s}^{+}$, while the variable $\boldsymbol{\varphi}^{-}=\alpha \boldsymbol{A}^{-} \eta^{\nabla}$ looks well suited for representing $\boldsymbol{P}_{s}^{-}$. But in our theory we want variables that are symmetric with respect to + and - strands, so the apparently unintuitive choice $\alpha \eta^{\nabla}=\frac{1}{2}\left(\varphi^{+}+\varphi^{-}\right)$is in fact natural, and yields compact expressions. The same remark holds true for the choice of alternative variables to parametrize $\boldsymbol{P}_{t}$. For the sake of uniformity of notation, we therefore introduce the following variables

$$
\begin{array}{ll}
\boldsymbol{v}^{\mathrm{c}}=\boldsymbol{w}^{\nabla}, & \boldsymbol{\gamma}^{\mathrm{c}}=\stackrel{\circ}{\boldsymbol{w}}, \\
\boldsymbol{u}^{\mathrm{c}}=\alpha \boldsymbol{\eta}^{\nabla}, & \boldsymbol{\omega}^{\mathrm{c}}=\alpha \stackrel{\circ}{\boldsymbol{\eta}},
\end{array}
$$

and refer to $\boldsymbol{v}^{\mathrm{c}}$ and $\boldsymbol{u}^{\mathrm{c}}$ as the microstrains, and $\boldsymbol{\gamma}^{\mathrm{c}}$ and $\boldsymbol{\omega}^{\mathrm{c}}$ as the microvelocities. 


\section{Balance laws}

In this section we establish the equations of motion for the birod by adding and subtracting the equations of motion of each of the individual strands.

\subsection{Balance of linear momentum}

Let $\boldsymbol{n}^{ \pm}(s, t)$ be the resultant forces (of the material with $\sigma>s$ on the material with $\sigma<s$ ) at time $t$ acting across the cross sections at $s$ of $\mathscr{R}^{ \pm}$. Similarly, let $\boldsymbol{p}^{ \pm}(s, t)$ be the densities of linear momenta of the two strands

$$
\boldsymbol{p}^{ \pm}(s, t):=\rho^{ \pm}(s) \boldsymbol{r}_{t}^{ \pm}(s, t) .
$$

In the absence of a distributed external force, the balance of linear momentum in $\mathscr{R}^{ \pm}$yields

$$
\boldsymbol{n}_{s}^{ \pm} \mp \boldsymbol{f}=\boldsymbol{p}_{t}^{ \pm}
$$

where $f(s, t)$ is the force (per unit length of $\widehat{\mathscr{C}}$ ) exerted by $\mathscr{R}^{+}$on $\mathscr{R}^{-}$. As stated earlier, in this section we shall assume that at each $s, \rho^{ \pm}(s)=\frac{1}{2} \lambda^{ \pm}(s) \rho(s)$ and that $\rho(s)=\rho^{+}(s)+\rho^{-}(s)$. Define

$$
\begin{aligned}
\boldsymbol{n}(s, t) & :=\boldsymbol{n}^{+}(s, t)+\boldsymbol{n}^{-}(s, t), \\
\boldsymbol{n}^{\mathrm{c}}(s, t) & :=\lambda^{-}(s) \boldsymbol{n}^{+}(s, t)-\lambda^{+}(s) \boldsymbol{n}^{-}(s, t),
\end{aligned}
$$

and

$$
\begin{aligned}
\boldsymbol{p}(s, t) & :=\boldsymbol{p}^{+}(s, t)+\boldsymbol{p}^{-}(s, t)=\rho(s) \boldsymbol{r}_{t}(s, t), \\
\boldsymbol{p}^{\mathrm{c}}(s, t) & :=\lambda^{-}(s) \boldsymbol{p}^{+}(s, t)-\lambda^{+}(s) \boldsymbol{p}^{-}(s, t)=\rho(s) \boldsymbol{w}_{t}(s, t) .
\end{aligned}
$$

Addition and weighted subtraction of the balance equations $\left(3.2^{ \pm}\right)$yields

$$
\begin{aligned}
\boldsymbol{n}_{s} & =\boldsymbol{p}_{t}, \\
\boldsymbol{n}_{s}^{\mathrm{c}}-\left(2 \boldsymbol{f}+\lambda_{s} \boldsymbol{n}\right) & =\boldsymbol{p}_{t}^{\mathrm{c}} .
\end{aligned}
$$

Note that while (3.5a) is the usual equation of balance of linear momentum of an elastic rod with no distributed forces, equation (3.5b) is of the form of the equation of balance of linear momentum of an elastic rod subject to distributed forces.

\subsection{Balance of angular momentum}

Let $\boldsymbol{m}^{ \pm}(s, t)$ be the resultant couples at time $t$ acting across the cross sections at $s$ of $\mathscr{R}^{ \pm}$about the points $\boldsymbol{r}^{ \pm}(s, t)$. Let $\pi^{ \pm}(s, t)$ be the densities of angular momenta at $s$ and at time $t$ of the two strands, so that

$$
\boldsymbol{\pi}^{ \pm}(s, t):=\boldsymbol{J}^{ \pm}(s) \boldsymbol{\omega}^{ \pm}(s, t),
$$

where $\boldsymbol{J}^{ \pm}(s)$ are the symmetric, positive-definite inertia tensors associated with the material cross sections at $s$ of the two strands. 
With use of $\left(2.12^{ \pm}\right)$and $\left(3.2^{ \pm}\right)$, and in the absence of a distributed external couple, balance of angular momentum (about the point $O$ ) of the strands $\mathscr{R}^{ \pm}$becomes

$$
\boldsymbol{m}_{s}^{ \pm}+\boldsymbol{r}_{s}^{ \pm} \times \boldsymbol{n}^{ \pm}+\boldsymbol{w} \times \boldsymbol{f} \mp \boldsymbol{c}=\boldsymbol{\pi}_{t}^{ \pm},
$$

where $\boldsymbol{c}(s, t)$ is the couple (per unit length of $\widehat{\mathscr{C}}$ ) exerted by $\mathscr{R}^{+}$on $\mathscr{R}^{-}$. Define

$$
\begin{aligned}
\boldsymbol{m}(s, t):= & \boldsymbol{m}^{+}(s, t)+\boldsymbol{m}^{-}(s, t)+\boldsymbol{w}(s, t) \times \boldsymbol{n}^{\mathrm{c}}(s, t), \\
\boldsymbol{m}^{\mathrm{c}}(s, t):=\lambda^{-}(s) \boldsymbol{m}^{+}(s, t)-\lambda^{+}(s) \boldsymbol{m}^{-}(s, t) & \\
& \quad+\boldsymbol{w}(s, t) \times\left(\left(\lambda^{-}(s)\right)^{2} \boldsymbol{n}^{+}(s, t)+\left(\lambda^{+}(s)\right)^{2} \boldsymbol{n}^{-}(s, t)\right),
\end{aligned}
$$

and

$$
\begin{aligned}
& \boldsymbol{\pi}(s, t):= \boldsymbol{\pi}^{+}(s, t)+\boldsymbol{\pi}^{-}(s, t)+\boldsymbol{w}(s, t) \times \boldsymbol{p}^{\mathrm{c}}(s, t) \\
& \boldsymbol{\pi}^{\mathrm{c}}(s, t):=\lambda^{-}(s) \boldsymbol{\pi}^{+}(s, t)-\lambda^{+}(s) \boldsymbol{\pi}^{-}(s, t) \\
& \quad+\boldsymbol{w}(s, t) \times\left(\left(\lambda^{-}(s)\right)^{2} \boldsymbol{p}^{+}(s, t)+\left(\lambda^{+}(s)\right)^{2} \boldsymbol{p}^{-}(s, t)\right) .
\end{aligned}
$$

We defer consideration of the expressions relating $\pi$ and $\pi^{\mathrm{c}}$ to the macroscopic and microscopic angular velocities until Section 6.

Addition and weighted subtraction of the balance equations $\left(3.7^{ \pm}\right)$and the use of (3.5b) yields

$$
\begin{aligned}
\boldsymbol{m}_{s}+\boldsymbol{r}_{s} \times \boldsymbol{n} & =\boldsymbol{\pi}_{t}, \\
\boldsymbol{m}_{s}^{\mathrm{c}}+\boldsymbol{r}_{s} \times \boldsymbol{n}^{\mathrm{c}}-2 \boldsymbol{c} & =\boldsymbol{\pi}_{t}^{\mathrm{c}}+\boldsymbol{r}_{t} \times \boldsymbol{p}^{\mathrm{c}} .
\end{aligned}
$$

Note that while (3.10a) is the usual equation of balance of angular momentum of an elastic rod with no distributed moments, equation (3.10b) is the equation of balance of angular momentum of an elastic rod with the same center line configuration, but with distributed moments. The extra term $\boldsymbol{r}_{t} \times \boldsymbol{p}^{\mathrm{c}}$ in (3.10b) arises from the fact that the linear momentum density $\boldsymbol{p}^{\mathrm{c}}$ is in general not parallel to $\boldsymbol{r}_{t}$. In (3.10a) the corresponding term vanishes because of the particular form (3.4a).

For later reference, we gather the balance equations of linear and angular momenta in the following system

$$
\begin{aligned}
\boldsymbol{n}_{s} & =\boldsymbol{p}_{t}, \\
\boldsymbol{m}_{s}+\boldsymbol{r}_{s} \times \boldsymbol{n} & =\boldsymbol{\pi}_{t}, \\
\boldsymbol{n}_{s}^{\mathrm{c}}-2 \boldsymbol{f} & =\boldsymbol{p}_{t}^{\mathrm{c}}, \\
\boldsymbol{m}_{s}^{\mathrm{c}}+\boldsymbol{r}_{s} \times \boldsymbol{n}^{\mathrm{c}}-2 \boldsymbol{c} & =\boldsymbol{\pi}_{t}^{\mathrm{c}}+\boldsymbol{r}_{t} \times \boldsymbol{p}^{\mathrm{c}} .
\end{aligned}
$$

These are the equations of motion of the birod. Equations $(3.11 \mathrm{a}, \mathrm{b})$ are the equations of macromotion, while equations $(3.11 \mathrm{c}, \mathrm{d})$ are the equations of micromotion.

For systems with one space dimension a balance law

$$
F_{s}=I_{t}
$$

is called a conservation law because, for suitable boundary conditions on the flux $F$, integration over the domain implies conservation of $\int I d s$. We can readily see that (3.11a) expresses conservation of linear momentum. By taking the cross product 
of $\boldsymbol{r}$ with (3.11a) and adding the resultant equation to (3.11b), we obtain the law of conservation of angular momentum

$$
(\boldsymbol{m}+\boldsymbol{r} \times \boldsymbol{n})_{s}=\boldsymbol{\pi}_{t} .
$$

We remark that, in general, equations $(3.11 \mathrm{c}, \mathrm{d})$ cannot be put into the form of conservation laws because of the additional terms. Nevertheless, in Section 5, we will show that equations (3.11) are the Euler-Lagrange equations of a Euclidean action principle.

\section{Constitutive relations}

Before discussing constitutive relations, we summarize the basic variables for the description of the dynamics of the composite birod $\mathscr{R}$. As noted earlier, there are basically two ways to describe the configuration of this composite structure made of two rods interacting elastically:

(i) as one elastic Cosserat rod endowed with two additional microstructure variables, i.e., as a birod.

(ii) as two special Cosserat rods which are bound together elastically, i.e., the two-rod description.

The configuration space for description (i) is

$$
\mathcal{C}:=\left\{(\boldsymbol{r}, \boldsymbol{R}, \boldsymbol{w}, \boldsymbol{P}):[0, L] \times[0, T) \mapsto \mathbb{R}^{3} \times S O(3) \times \mathbb{R}^{3} \times S O(3)\right\},
$$

while the configuration space for description (ii) is $\mathcal{C}^{+} \times \mathcal{C}^{-}$where

$$
\mathcal{C}^{ \pm}:=\left\{\left(\boldsymbol{r}^{ \pm}, \boldsymbol{R}^{ \pm}\right):[0, L] \times[0, T) \mapsto \mathbb{R}^{3} \times S O(3)\right\}
$$

Thus the configuration spaces for both descriptions are equivalent.

In Box 1 we give a mapping which expresses the basic variables of the birod description (i) in terms of the basic variables of the two-rod description (ii), and in Box 3 we give the inverse mapping, i.e., we express the basic variables of description (ii) in terms of the basic variables of the description (i). In Boxes 2 and 4 we present the balance laws in the two-rod description and the birod description respectively. It remains to postulate constitutive relations for the birod, which may be done in either of the two different ways: we may postulate these relations in terms of the basic variables of description (ii), and then derive constitutive relations for description (i), or we may postulate constitutive relations in terms of the basic variables of description (i), and use them to obtain the constitutive relations for description (ii). In Section 5 we will use an action principle to systematically obtain the equations of motion and hyper-elastic constitutive relations for the birod using the second approach. For simplicity, in that section we shall assume that the strands have equal mass densities, i.e., $\rho^{+}=\rho^{-}=\frac{1}{2} \rho$, or, equivalently $\lambda^{ \pm} \equiv 1$. 
$\left.\begin{array}{l}\boldsymbol{r} \\ \boldsymbol{w} \\ \boldsymbol{R} \\ \boldsymbol{P}\end{array}\right\} \longrightarrow\left\{\begin{array}{l}\boldsymbol{r}^{+}=\boldsymbol{r}+\lambda^{-} \boldsymbol{w} \\ \boldsymbol{r}^{-}=\boldsymbol{r}-\lambda^{+} \boldsymbol{w} \\ \boldsymbol{R}^{+}=\boldsymbol{P} \boldsymbol{R} \\ \boldsymbol{R}^{-}=\boldsymbol{P}^{T} \boldsymbol{R}\end{array}\right.$

Configuration

$\left.\begin{array}{l}\boldsymbol{v} \\ \boldsymbol{w}_{s} \\ \boldsymbol{\gamma} \\ \boldsymbol{w}_{t} \\ \boldsymbol{u} \\ \boldsymbol{u}^{\mathrm{c}} \\ \boldsymbol{\omega} \\ \boldsymbol{\omega}^{\mathrm{c}}\end{array}\right\} \longrightarrow\left\{\begin{array}{l}\boldsymbol{v}^{+}=\left[\boldsymbol{v}+\lambda^{-} \boldsymbol{w}_{s}+\lambda_{s}^{-} \boldsymbol{w}\right] / g^{+} \\ \boldsymbol{v}^{-}=\left[\boldsymbol{v}-\lambda^{+} \boldsymbol{w}_{s}+\lambda_{s}^{-} \boldsymbol{w}\right] / g^{-} \\ \boldsymbol{\gamma}^{+}=\boldsymbol{\gamma}+\lambda^{-} \boldsymbol{w}_{t} \\ \boldsymbol{\gamma}^{-}=\boldsymbol{\gamma}-\lambda^{+} \boldsymbol{w}_{t} \\ \boldsymbol{u}^{+}=\left[\boldsymbol{u}+\boldsymbol{A} \boldsymbol{u}^{\mathrm{c}}\right] / g^{+} \\ \boldsymbol{u}^{-}=\left[\boldsymbol{u}-\boldsymbol{A}^{T} \boldsymbol{u}^{\mathrm{c}}\right] / g^{-} \\ \omega^{+}=\boldsymbol{\omega}+\boldsymbol{A} \boldsymbol{\omega}^{\mathrm{c}} \\ \omega^{-}=\boldsymbol{\omega}-\boldsymbol{A}^{T} \boldsymbol{\omega}^{\mathrm{c}}\end{array}\right.$

Kinematics

of strains

$\left.\begin{array}{l}\boldsymbol{n} \\ \boldsymbol{n}^{\mathrm{c}} \\ \boldsymbol{p} \\ \boldsymbol{p}^{\mathrm{c}} \\ \boldsymbol{m} \\ \boldsymbol{m}^{\mathrm{c}} \\ \boldsymbol{\pi} \\ \boldsymbol{\pi}^{\mathrm{c}} \\ \boldsymbol{f} \\ \boldsymbol{c}\end{array}\right\} \longrightarrow\left\{\begin{array}{l}\boldsymbol{n}^{+}=\frac{1}{2}\left[\lambda^{+} \boldsymbol{n}+\boldsymbol{n}^{\mathrm{c}}\right] \\ \boldsymbol{n}^{-}=\frac{1}{2}\left[\lambda^{-} \boldsymbol{n}-\boldsymbol{n}^{\mathrm{c}}\right] \\ \boldsymbol{p}^{+}=\frac{1}{2}\left[\lambda^{+} \boldsymbol{p}+\boldsymbol{p}^{\mathrm{c}}\right] \\ \boldsymbol{p}^{-}=\frac{1}{2}\left[\lambda^{-} \boldsymbol{p}-\boldsymbol{p}^{\mathrm{c}}\right] \\ \boldsymbol{m}^{+}=\frac{1}{2}\left[\lambda^{+} \boldsymbol{m}+\boldsymbol{m}^{\mathrm{c}}-\lambda^{-} \boldsymbol{w} \times\left(\lambda^{+} \boldsymbol{n}+\boldsymbol{n}^{\mathrm{c}}\right)\right] \\ \boldsymbol{m}^{-}=\frac{1}{2}\left[\lambda^{-} \boldsymbol{m}-\boldsymbol{m}^{\mathrm{c}}+\lambda^{+} \boldsymbol{w} \times\left(\lambda^{-} \boldsymbol{n}-\boldsymbol{n}^{\mathrm{c}}\right)\right] \\ \boldsymbol{\pi}^{+}=\frac{1}{2}\left[\lambda^{+} \boldsymbol{\pi}+\boldsymbol{\pi}^{\mathrm{c}}-\lambda^{-} \boldsymbol{w} \times\left(\lambda^{+} \boldsymbol{p}+\boldsymbol{p}^{\mathrm{c}}\right)\right] \\ \boldsymbol{\pi}^{-}=\frac{1}{2}\left[\lambda^{-} \boldsymbol{\pi}-\boldsymbol{\pi}^{\mathrm{c}}+\lambda^{+} \boldsymbol{w} \times\left(\lambda^{-} \boldsymbol{p}-\boldsymbol{p}^{\mathrm{c}}\right)\right] \\ \boldsymbol{f} \\ \boldsymbol{c}\end{array}\right.$

Stresses

where

$g^{+}=\left\|\widehat{\boldsymbol{r}}_{s}+\lambda^{-} \widehat{\boldsymbol{w}}_{s}+\lambda_{s}^{-} \widehat{\boldsymbol{w}}\right\|, \quad g^{-}=\left\|\widehat{\boldsymbol{r}}_{s}-\lambda^{+} \widehat{\boldsymbol{w}}_{s}+\lambda_{s}^{-} \widehat{\boldsymbol{w}}\right\|$,

$\boldsymbol{A}=\boldsymbol{I}+\boldsymbol{\eta}^{\times}$, and $\boldsymbol{\eta}$ is the Gibbs rotation vector of $\boldsymbol{P}$.

Box 1. Transformation from birod to two-rod description.

$$
\begin{aligned}
& \boldsymbol{n}_{s}^{+}-\boldsymbol{f}=\boldsymbol{p}_{t}^{+} \\
& \boldsymbol{m}_{s}^{+}+\boldsymbol{r}_{s}^{+} \times \boldsymbol{n}^{+}+\frac{1}{2}\left(\boldsymbol{r}^{+}-\boldsymbol{r}^{-}\right) \times \boldsymbol{f}-\boldsymbol{c}=\boldsymbol{\pi}_{t}^{+}
\end{aligned} \quad \quad \text { Balance laws for } \mathscr{R}^{+}
$$

Box 2. Balance laws for the two-rod description. 
$\left.\begin{array}{l}\boldsymbol{r}^{+} \\ \boldsymbol{r}^{-} \\ \boldsymbol{R}^{+} \\ \boldsymbol{R}^{-}\end{array}\right\} \rightarrow\left\{\begin{array}{l}\boldsymbol{r}=\frac{1}{2}\left[\lambda^{+} \boldsymbol{r}^{+}+\lambda^{-} \boldsymbol{r}^{-}\right] \\ \boldsymbol{w}=\frac{1}{2}\left[\boldsymbol{r}^{+}-\boldsymbol{r}^{-}\right] \\ \boldsymbol{R}=\left[\boldsymbol{R}^{+} \boldsymbol{R}^{-T}\right]^{1 / 2} \boldsymbol{R}^{-} \\ \boldsymbol{P}=\left[\boldsymbol{R}^{+} \boldsymbol{R}^{-T}\right]^{1 / 2}\end{array}\right.$

Configuration

$\left.\begin{array}{rl}\boldsymbol{v}^{+} \\ \boldsymbol{v}^{-} \\ \boldsymbol{\gamma}^{+} \\ \boldsymbol{\gamma}^{-} \\ \boldsymbol{u}^{+} \\ \boldsymbol{u}^{-} \\ \boldsymbol{\omega}^{+} \\ \boldsymbol{\omega}^{-}\end{array}\right\} \rightarrow\left\{\begin{aligned} \boldsymbol{v} & =\frac{1}{2}\left[h^{+}\left(\lambda^{+} \boldsymbol{v}^{+}+\lambda_{s}^{+} \boldsymbol{r}^{+}\right)+h^{-}\left(\lambda^{-} \boldsymbol{v}^{-}+\lambda_{s}^{-} \boldsymbol{r}^{-}\right)\right] \\ \boldsymbol{w}_{s} & =\frac{1}{2}\left[h^{+} \boldsymbol{v}^{+}-h^{-} \boldsymbol{v}^{-}\right] \\ \boldsymbol{\gamma} & =\frac{1}{2}\left[\boldsymbol{\gamma}^{+}+\boldsymbol{\gamma}^{-}\right] \\ \boldsymbol{w}_{t} & =\frac{1}{2}\left[\boldsymbol{\gamma}^{+}-\boldsymbol{\gamma}^{-}\right] \\ \boldsymbol{u} & =\frac{1}{2}\left[h^{+} \boldsymbol{B}^{T} \boldsymbol{u}^{+}+h^{-} \boldsymbol{B} \boldsymbol{u}^{-}\right] \\ \boldsymbol{u}^{\mathrm{c}} & =\frac{1}{2}\left[h^{+} \boldsymbol{u}^{+}-h^{-} \boldsymbol{u}^{-}\right] \\ \boldsymbol{\omega} & =\frac{1}{2}\left[\boldsymbol{B}^{T} \boldsymbol{\omega}^{+}+\boldsymbol{B} \boldsymbol{\omega}^{-}\right] \\ \boldsymbol{\omega}^{\mathrm{c}} & =\frac{1}{2}\left[\boldsymbol{\omega}^{+}-\boldsymbol{\omega}^{-}\right]\end{aligned}\right.$

Kinematics

of strains

$\left.\begin{array}{rl}n^{+} \\ n^{-} \\ p^{+} \\ p^{-}\end{array}\right) \quad\left(\begin{array}{rl}n & =n^{+}+n^{-} \\ n^{\mathrm{c}} & =\lambda^{-} n^{+}-\lambda^{+} n^{-} \\ p & =p^{+}+p^{-} \\ p^{\mathrm{c}} & =\lambda^{-} p^{+}-\lambda^{+} p^{-}\end{array}\right.$

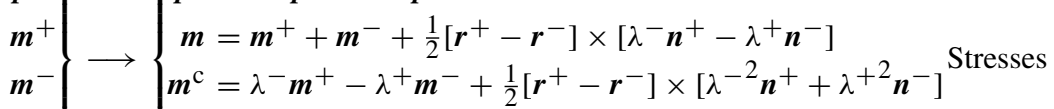

$\boldsymbol{\pi}^{+} \quad \boldsymbol{\pi}=\boldsymbol{\pi}^{+}+\boldsymbol{\pi}^{-}+\frac{1}{2}\left[\boldsymbol{r}^{+}-\boldsymbol{r}^{-}\right] \times\left[\lambda^{-} \boldsymbol{p}^{+}-\lambda^{+} \boldsymbol{p}^{-}\right]$

$\pi^{-}$
$f$$\quad \quad \begin{aligned} & \pi^{\mathrm{c}}=\lambda^{-} \pi^{+}-\lambda^{+} \pi^{-}+\frac{1}{2}\left[\boldsymbol{r}^{+}-\boldsymbol{r}^{-}\right] \times\left[\lambda^{-2} p^{+}+\lambda^{+2} p^{-}\right] \\ & \boldsymbol{f} \\ & c\end{aligned}$

where

$h^{+}=\left\|\widehat{\boldsymbol{r}}_{S}^{+}\right\|, \quad h^{-}=\left\|\widehat{\boldsymbol{r}}_{s}^{-}\right\|$,

$\boldsymbol{B}=\boldsymbol{I}+\zeta^{\times}, \quad$ and $\zeta$ is the Gibbs rotation vector of $\left[\boldsymbol{R}^{+} \boldsymbol{R}^{-T}\right]^{1 / 2}$.

Box 3. Transformation from two-rod to birod description.

$$
\begin{array}{l|l}
\begin{array}{l}
\boldsymbol{n}_{s}=\boldsymbol{p}_{t} \\
\boldsymbol{m}_{S}+\boldsymbol{r}_{s} \times \boldsymbol{n}=\boldsymbol{\pi}_{t}
\end{array} & \text { Macroscopic balance laws } \\
\left.\begin{array}{l}
\boldsymbol{n}_{s}^{\mathrm{c}}-\left(2 \boldsymbol{f}+\lambda_{s}^{-} \boldsymbol{n}\right)=\boldsymbol{p}_{t}^{\mathrm{c}} \\
\boldsymbol{m}_{s}^{\mathrm{c}}+\boldsymbol{r}_{s} \times \boldsymbol{n}^{\mathrm{c}}-\left(2 \boldsymbol{c}+\lambda_{s}^{-} \boldsymbol{m}\right)=\boldsymbol{\pi}_{t}^{\mathrm{c}}+\boldsymbol{r}_{t} \times \boldsymbol{p}^{\mathrm{c}}
\end{array}\right\} \text { Microscopic balance laws }
\end{array}
$$

Box 4. Balance laws for the birod. 


\section{Variational formulation}

\subsection{Action density for a rod with microstructure}

We consider a special Cosserat rod $\mathscr{R}$ endowed with two microstructure variables: a vector $\boldsymbol{w}$ and a rotation tensor $\boldsymbol{P}$. Here $\boldsymbol{w}$ is a prototype of a vector and $\boldsymbol{P}$ is a prototype of a rotation tensor. In the following analysis, we could include as many variables of both types as we wish, but we explicitly consider only the case of one of each type as it is the case pertinent for birods.

Let $\mathscr{P}$ denote the line segment $s_{1} \leqq s \leqq s_{2}$ and let $\mathscr{I}$ be the time interval $t_{1} \leqq t \leqq t_{2}$. With every set $\mathscr{P} \times \mathscr{I}$ we associate the action $\mathcal{A}$ given by

$$
\mathcal{A}=\int_{t_{1}}^{t_{2}} \int_{s_{1}}^{s_{2}} \widetilde{L}\left(s, t ; \boldsymbol{r}, \boldsymbol{r}_{s}, \boldsymbol{r}_{t}, \boldsymbol{R}, \boldsymbol{R}_{s}, \boldsymbol{R}_{t}, \boldsymbol{w}, \boldsymbol{w}_{s}, \boldsymbol{w}_{t}, \boldsymbol{P}, \boldsymbol{P}_{s}, \boldsymbol{P}_{t}\right) d s d t .
$$

Here the action density $\widetilde{\mathfrak{L}}$ is a function of $s, t$ and the macroscopic and microscopic kinematical variables as well as their spatial and time derivatives.

As mentioned at the end of Section 2, it is more convenient to work with the convected forms (as defined in (2.38)) of the microstrains $\boldsymbol{v}^{\mathrm{c}}:=\boldsymbol{w}^{\nabla}$ and $\boldsymbol{\gamma}^{\mathrm{c}}:=\stackrel{\circ}{\boldsymbol{w}}$ instead of the space and time derivatives $\boldsymbol{w}_{s}$ and $\boldsymbol{w}_{t}$. There is no need to parametrize the rotation tensor $\boldsymbol{R}$ because frame indifference will imply that the action density has no explicit dependence on it. In contrast, we do need to parametrize the rotation tensor $\boldsymbol{P}$. The final equations of motion we obtain from an action principle are equivalent whatever choice of variables we use to represent the spatial and temporal rates of rotation of $\boldsymbol{P}$. Nevertheless, the most suitable choice is problematic, and it seems that there is no choice that systematically and directly yields the final form of the equation of macromoment derived below. We proceed with the choice $\boldsymbol{\eta}, \boldsymbol{u}^{\mathrm{c}}:=\alpha \boldsymbol{\eta}^{\nabla}$ and $\boldsymbol{\omega}^{\mathrm{c}}:=\alpha \stackrel{\circ}{\boldsymbol{\eta}}$ instead of $\boldsymbol{P}, \boldsymbol{P}_{s}$ and $\boldsymbol{P}_{t}$, where, as before, $\boldsymbol{\eta}$ is the Gibbs rotation vector associated with the rotation tensor $\boldsymbol{P}$, and $\alpha=2 /(1+\boldsymbol{\eta} \cdot \boldsymbol{\eta})$. Accordingly, we introduce a new action density $\mathfrak{L}$ by

$$
\begin{aligned}
\mathfrak{L}\left(s, t ; \boldsymbol{r}, \boldsymbol{r}_{s}, \boldsymbol{r}_{t}, \boldsymbol{R}, \boldsymbol{R}_{s}, \boldsymbol{R}_{t}, \boldsymbol{w}, \boldsymbol{v}^{\mathrm{c}}, \boldsymbol{\gamma}^{\mathrm{c}}, \boldsymbol{\eta}, \boldsymbol{u}^{\mathrm{c}}, \omega^{\mathrm{c}}\right) \\
\quad:=\widetilde{\mathfrak{L}}\left(s, t ; \boldsymbol{r}, \boldsymbol{r}_{s}, \boldsymbol{r}_{t}, \boldsymbol{R}, \boldsymbol{R}_{s}, \boldsymbol{R}_{t}, \boldsymbol{w}, \boldsymbol{w}_{s}, \boldsymbol{w}_{t}, \boldsymbol{P}, \boldsymbol{P}_{s}, \boldsymbol{P}_{t}\right) .
\end{aligned}
$$

\subsection{Invariance under Euclidean displacements}

Following the pioneering work of the COSSERAT brothers [11], we adopt invariance of the action density under Euclidean displacements as a principle, see for example [26, 29]. This is a weaker condition than the principle of material frame indifference that is often adopted in classical continuum mechanics, but, as was pointed out by TRUESDELL [30], it suffices for elasticity theory.

We now examine the effects of a Euclidean displacement characterized by a constant orthogonal tensor $\boldsymbol{Q}$ and a constant translation vector $\boldsymbol{b}$ along with a time shift $t^{\sharp}=t+\varpi$. As we can see, this is a special change of frame where the rotation tensor and the translation vector are independent of time. 
In the remainder of this article, quantities associated with the configuration after a Euclidean displacement will be denoted by a superposed $\sharp$. The center line $\boldsymbol{r}(s, t)$, and its spatial and time derivatives transform to

$$
\begin{aligned}
\boldsymbol{r}^{\sharp}\left(s, t^{\sharp}\right) & =\boldsymbol{Q} \boldsymbol{r}(s, t)+\boldsymbol{b}, \\
\boldsymbol{r}_{s}^{\sharp}\left(s, t^{\sharp}\right) & =\boldsymbol{Q} \boldsymbol{r}_{s}(s, t), \\
\boldsymbol{r}_{t^{\sharp}}^{\sharp}\left(s, t^{\sharp}\right) & =\boldsymbol{Q} \boldsymbol{r}_{t}(s, t) .
\end{aligned}
$$

As the directors $\boldsymbol{d}_{i}(s, t)$ transform to

$$
\boldsymbol{d}_{i}^{\sharp}\left(s, t^{\sharp}\right)=\boldsymbol{Q} \boldsymbol{d}_{i}(s, t),
$$

it follows that $\boldsymbol{R}(s, t), \boldsymbol{R}_{S}(s, t)$ and $\boldsymbol{R}_{t}(s, t)$ transform to

$$
\begin{aligned}
\boldsymbol{R}^{\sharp}\left(s, t^{\sharp}\right) & =\boldsymbol{Q} \boldsymbol{R}(s, t), \\
\boldsymbol{R}_{s}^{\sharp}\left(s, t^{\sharp}\right) & =\boldsymbol{Q} \boldsymbol{R}_{s}(s, t), \\
\boldsymbol{R}_{t^{\sharp}}^{\sharp}\left(s, t^{\sharp}\right) & =\boldsymbol{Q} \boldsymbol{R}_{t}(s, t),
\end{aligned}
$$

from which we conclude that $\boldsymbol{u}(s, t)$ and $\boldsymbol{\omega}(s, t)$ transform as

$$
\begin{aligned}
& \boldsymbol{u}^{\sharp}\left(s, t^{\sharp}\right)=\boldsymbol{Q} \boldsymbol{u}(s, t), \\
& \boldsymbol{\omega}^{\sharp}\left(s, t^{\sharp}\right)=\boldsymbol{Q} \boldsymbol{\omega}(s, t) .
\end{aligned}
$$

The microstructure vectors $\boldsymbol{w}(s, t)$ and $\boldsymbol{\eta}(s, t)$ transform to

$$
\begin{aligned}
\boldsymbol{w}^{\sharp}\left(s, t^{\sharp}\right) & =\boldsymbol{Q} \boldsymbol{w}(s, t), \\
\eta^{\sharp}\left(s, t^{\sharp}\right) & =\boldsymbol{Q} \boldsymbol{\eta}(s, t) .
\end{aligned}
$$

From (5.6), (5.7) and the definitions (2.51) we obtain

$$
\begin{aligned}
\boldsymbol{v}^{c \sharp}\left(s, t^{\sharp}\right)=\boldsymbol{Q} \boldsymbol{v}^{\mathrm{c}}(s, t), & \boldsymbol{\gamma}^{c \sharp}\left(s, t^{\sharp}\right)=\boldsymbol{Q} \boldsymbol{\gamma}^{\mathrm{c}}(s, t), \\
\boldsymbol{u}^{c \sharp}\left(s, t^{\sharp}\right)=\boldsymbol{Q} \boldsymbol{u}^{\mathrm{c}}(s, t), & \boldsymbol{\omega}^{c \sharp}\left(s, t^{\sharp}\right)=\boldsymbol{Q} \boldsymbol{\omega}^{\mathrm{c}}(s, t) .
\end{aligned}
$$

For the action density $\mathfrak{L}$ to be invariant under the group of Euclidean displacements $\mathfrak{L}$ must satisfy

$$
\begin{aligned}
\mathfrak{L}\left(s, t^{\sharp} ; \boldsymbol{r}^{\sharp}, \boldsymbol{r}_{s}^{\sharp}, \boldsymbol{r}_{t}^{\sharp}, \boldsymbol{R}^{\sharp}, \boldsymbol{R}_{s}^{\sharp}, \boldsymbol{R}_{t}^{\sharp}, \boldsymbol{w}^{\sharp}, \boldsymbol{v}^{c \sharp}, \boldsymbol{\gamma}^{c \sharp}, \boldsymbol{\eta}^{\sharp}, \boldsymbol{u}^{c \sharp}, \boldsymbol{\omega}^{c \sharp}\right) \\
=\mathfrak{L}\left(s, t ; \boldsymbol{r}, \boldsymbol{r}_{s}, \boldsymbol{r}_{t}, \boldsymbol{R}, \boldsymbol{R}_{s}, \boldsymbol{R}_{t}, \boldsymbol{w}, \boldsymbol{v}^{\mathrm{c}}, \boldsymbol{\gamma}^{\mathrm{c}}, \boldsymbol{\eta}, \boldsymbol{u}^{\mathrm{c}}, \boldsymbol{\omega}^{\mathrm{c}}\right) .
\end{aligned}
$$

Since the group of Euclidean displacements is a connected Lie group, to obtain the necessary conditions for such invariance it suffices to consider infinitesimal transformations [29]. It follows that $\mathfrak{L}$ is invariant under the group of Euclidean displacements if and only if

$$
\frac{\partial \mathfrak{L}}{\partial t}=0, \quad \frac{\partial \mathfrak{L}}{\partial \boldsymbol{r}}=\mathbf{0}, \quad \boldsymbol{M}-\boldsymbol{M}^{T}=\mathbf{0},
$$


where the second order tensor $\boldsymbol{M}$ is

$$
\begin{aligned}
\boldsymbol{M}= & \boldsymbol{r}_{s} \otimes \frac{\partial \mathfrak{L}}{\partial \boldsymbol{r}_{s}}+\boldsymbol{r}_{t} \otimes \frac{\partial \mathfrak{L}}{\partial \boldsymbol{r}_{t}}+\boldsymbol{R}\left(\frac{\partial \mathfrak{L}}{\partial \boldsymbol{R}}\right)^{T}+\boldsymbol{R}_{s}\left(\frac{\partial \mathfrak{L}}{\partial \boldsymbol{R}_{s}}\right)^{T}+\boldsymbol{R}_{t}\left(\frac{\partial \mathfrak{L}}{\partial \boldsymbol{R}_{t}}\right)^{T} \\
& +\boldsymbol{w} \otimes \frac{\partial \mathfrak{L}}{\partial \boldsymbol{w}}+\boldsymbol{v}^{\mathrm{c}} \otimes \frac{\partial \mathfrak{L}}{\partial \boldsymbol{v}^{\mathrm{c}}}+\boldsymbol{\gamma}^{\mathrm{c}} \otimes \frac{\partial \mathfrak{L}}{\partial \boldsymbol{\gamma}^{\mathrm{c}}}+\boldsymbol{\eta} \otimes \frac{\partial \mathfrak{L}}{\partial \boldsymbol{\eta}}+\boldsymbol{u}^{\mathrm{c}} \otimes \frac{\partial \mathfrak{L}}{\partial \boldsymbol{u}^{\mathrm{c}}}+\boldsymbol{\omega}^{\mathrm{c}} \otimes \frac{\partial \mathfrak{L}}{\partial \boldsymbol{\omega}^{\mathrm{c}}} .
\end{aligned}
$$

Conditions (5.10) $)_{1}$ and (5.10) 2 imply that $\mathfrak{L}$ must be independent of $t$ and $\boldsymbol{r}$, which we accordingly drop from the list of arguments. Note that conditions $(5.10)_{2}$ and $(5.10)_{3}$ express conservation of linear and angular momenta.

To get the appropriately reduced form of the action density we introduce the reduced variables

$$
\begin{array}{rlrlrl}
\mathbf{v} & =\boldsymbol{R}^{T} \boldsymbol{r}_{s}, & \boldsymbol{\gamma} & =\boldsymbol{R}^{T} \boldsymbol{r}_{t}, \\
\mathbf{u}^{\times} & =\boldsymbol{R}^{T} \boldsymbol{R}_{s}, & \boldsymbol{\omega}^{\times} & =\boldsymbol{R}^{T} \boldsymbol{R}_{t}, \\
\mathbf{w}=\boldsymbol{R}^{T} \boldsymbol{w}, & \mathbf{v}^{\mathrm{c}} & =\boldsymbol{R}^{T} \boldsymbol{v}^{\mathrm{c}}, & \boldsymbol{\gamma}^{\mathrm{c}} & =\boldsymbol{R}^{T} \boldsymbol{\gamma}^{\mathrm{c}}, \\
\boldsymbol{\eta}=\boldsymbol{R}^{T} \boldsymbol{\eta}, & \mathbf{u}^{\mathrm{c}} & =\boldsymbol{R}^{T} \boldsymbol{u}^{\mathrm{c}}, & \boldsymbol{\omega}^{\mathrm{c}} & =\boldsymbol{R}^{T} \boldsymbol{\omega}^{\mathrm{c}},
\end{array}
$$

and the reduced function

$$
\begin{aligned}
\mathcal{L}\left(s ; \boldsymbol{R}, \mathbf{v}, \boldsymbol{\gamma}, \mathbf{u}, \boldsymbol{\omega}, \mathbf{w}, \mathbf{v}^{\mathrm{c}}, \boldsymbol{\gamma}^{\mathrm{c}}, \boldsymbol{\eta}, \mathbf{u}^{\mathrm{c}}, \boldsymbol{\omega}^{\mathrm{c}}\right) \\
\quad:=\mathfrak{L}\left(s ; \boldsymbol{r}_{s}, \boldsymbol{r}_{t}, \boldsymbol{R}, \boldsymbol{R}_{s}, \boldsymbol{R}_{t}, \boldsymbol{w}, \boldsymbol{v}^{\mathrm{c}}, \boldsymbol{\gamma}^{\mathrm{c}}, \boldsymbol{\eta}, \boldsymbol{u}^{\mathrm{c}}, \boldsymbol{\omega}^{\mathrm{c}}\right) .
\end{aligned}
$$

Of course, because $\mathbf{v} \cdot \boldsymbol{e}_{i}=\boldsymbol{R}^{T} \boldsymbol{v} \cdot \boldsymbol{e}_{i}=\boldsymbol{v} \cdot \boldsymbol{R} \boldsymbol{e}_{i}=\boldsymbol{v} \cdot \boldsymbol{d}_{i}$, the components of the vector $\mathbf{v}$ with respect to the fixed frame $\left\{\boldsymbol{e}_{i}\right\}$ coincide with the components of the vector $\boldsymbol{v}$ with respect to the moving frame $\left\{\boldsymbol{d}_{i}\right\}$. Similarly, the components in the fixed frame $\left\{\boldsymbol{e}_{i}\right\}$ of the vectors $\boldsymbol{\gamma}, \mathbf{u}, \boldsymbol{\omega}, \mathbf{w}, \mathbf{v}^{\mathrm{c}}, \boldsymbol{\gamma}^{\mathrm{c}}, \boldsymbol{\eta}, \mathbf{u}^{\mathrm{c}}$, and $\boldsymbol{\omega}^{\mathrm{c}}$, coincide with the components in the moving frame $\left\{\boldsymbol{d}_{i}\right\}$ of the vectors $\boldsymbol{\gamma}, \boldsymbol{u}, \boldsymbol{\omega}, \boldsymbol{w}, \boldsymbol{v}^{\mathrm{c}}, \boldsymbol{\gamma}^{\mathrm{c}}, \boldsymbol{\eta}, \boldsymbol{u}^{\mathrm{c}}$, and $\omega^{\mathrm{c}}$, respectively.

Then, using (5.10) 1 and (5.10) 2 in (5.9) and noting that $\mathbf{v}^{\sharp}:=\boldsymbol{R}^{\sharp^{T}} \boldsymbol{r}_{S}^{\sharp}=$ $\boldsymbol{R}^{T} \boldsymbol{Q}^{T} \boldsymbol{Q} \boldsymbol{r}_{s}=\boldsymbol{R}^{T} \boldsymbol{r}_{s}=\mathbf{v}$, etc., we conclude that the functional equation

$$
\begin{aligned}
& \mathcal{L}\left(s ; \boldsymbol{R}, \mathbf{v}, \boldsymbol{\gamma}, \mathbf{u}, \boldsymbol{\omega}, \mathbf{w}, \mathbf{v}^{\mathrm{c}}, \boldsymbol{\gamma}^{\mathrm{c}}, \boldsymbol{\eta}, \mathbf{u}^{\mathrm{c}}, \boldsymbol{\omega}^{\mathrm{c}}\right) \\
& \quad=\mathcal{L}\left(s ; \boldsymbol{Q} \boldsymbol{R}, \mathbf{v}, \boldsymbol{\gamma}, \mathbf{u}, \boldsymbol{\omega}, \mathbf{w}, \mathbf{v}^{\mathrm{c}}, \boldsymbol{\gamma}^{\mathrm{c}}, \boldsymbol{\eta}, \mathbf{u}^{\mathrm{c}}, \boldsymbol{\omega}^{\mathrm{c}}\right),
\end{aligned}
$$

must hold for all values of the arguments in the domain of $\mathcal{L}$ and for all orthogonal tensors $\boldsymbol{Q}$. Therefore, $\mathcal{L}$ must be independent of $\boldsymbol{R}$, which will be dropped from the list of arguments, and hence the final reduced form of the action density is

$$
\begin{aligned}
\mathcal{L}\left(s ; \mathbf{v}, \boldsymbol{\gamma}, \mathbf{u}, \boldsymbol{\omega}, \mathbf{w}, \mathbf{v}^{\mathrm{c}}, \boldsymbol{\gamma}^{\mathrm{c}}, \boldsymbol{\eta}, \mathbf{u}^{\mathrm{c}}, \boldsymbol{\omega}^{\mathrm{c}}\right) \\
\quad:=\mathfrak{L}\left(s ; \boldsymbol{r}_{s}, \boldsymbol{r}_{t}, \boldsymbol{R}, \boldsymbol{R}_{s}, \boldsymbol{R}_{t}, \boldsymbol{w}, \boldsymbol{v}^{\mathrm{c}}, \boldsymbol{\gamma}^{\mathrm{c}}, \boldsymbol{\eta}, \boldsymbol{u}^{\mathrm{c}}, \boldsymbol{\omega}^{\mathrm{c}}\right) .
\end{aligned}
$$

To proceed further, we assume that $\mathcal{L}$ has the separated form

$$
\begin{aligned}
\mathcal{L}\left(s ; \mathbf{v}, \boldsymbol{\gamma}, \mathbf{u}, \boldsymbol{\omega}, \mathbf{w}, \mathbf{v}^{\mathrm{c}}, \boldsymbol{\gamma}^{\mathrm{c}}, \boldsymbol{\eta}, \mathbf{u}^{\mathrm{c}}, \boldsymbol{\omega}^{\mathrm{c}}\right) \\
\quad=\mathcal{K}\left(s ; \boldsymbol{\gamma}, \boldsymbol{\omega}, \mathbf{w}, \boldsymbol{\gamma}^{\mathrm{c}}, \boldsymbol{\eta}, \boldsymbol{\omega}^{\mathrm{c}}\right)-\mathcal{W}\left(s ; \mathbf{v}, \mathbf{u}, \mathbf{w}, \mathbf{v}^{\mathrm{c}}, \boldsymbol{\eta}, \mathbf{u}^{\mathrm{c}}\right),
\end{aligned}
$$

where $\mathcal{K}$ is the kinetic energy density and $\mathcal{W}$ is the strain energy density. It is usually assumed that the kinetic energy is a quadratic form in velocities, but we do not make such an assumption at this point (see the remark at the end of Section 5.4 below). 


\subsection{Hamilton's principle}

According to Hamilton's principle the equations of motion for a rod with the action given in (5.12) should coincide with Euler-Lagrange equations that have the weak variational form

$$
\delta \mathcal{A}(\mathscr{P} \times \mathscr{I})=0
$$

with respect to the independent field variables $\boldsymbol{r}, \boldsymbol{R}, \boldsymbol{w}$ and $\boldsymbol{\eta}$. With (5.12), the variational equation (5.13) becomes

$$
\begin{aligned}
\int_{t_{1}}^{t_{2}} \int_{s_{1}}^{s_{2}} & {\left[\frac{\partial \mathcal{K}}{\partial \boldsymbol{\gamma}} \cdot \delta \boldsymbol{\gamma}+\frac{\partial \mathcal{K}}{\partial \boldsymbol{\omega}} \cdot \delta \boldsymbol{\omega}+\frac{\partial \mathcal{K}}{\partial \mathbf{w}} \cdot \delta \mathbf{w}+\frac{\partial \mathcal{K}}{\partial \boldsymbol{\gamma}^{\mathrm{c}}} \cdot \delta \boldsymbol{\gamma}^{\mathrm{c}}\right.} \\
& +\frac{\partial \mathcal{K}}{\partial \boldsymbol{\eta}} \cdot \delta \boldsymbol{\eta}+\frac{\partial \mathcal{K}}{\partial \boldsymbol{\omega}^{\mathrm{c}}} \cdot \delta \boldsymbol{\omega}^{\mathrm{c}}-\frac{\partial \mathcal{W}}{\partial \mathbf{v}} \cdot \delta \mathbf{v}-\frac{\partial \mathcal{W}}{\partial \mathbf{u}} \cdot \delta \mathbf{u} \\
& \left.-\frac{\partial \mathcal{W}}{\partial \mathbf{w}} \cdot \delta \mathbf{w}-\frac{\partial \mathcal{W}}{\partial \mathbf{v}^{\mathrm{c}}} \cdot \delta \mathbf{v}^{\mathrm{c}}-\frac{\partial \mathcal{W}}{\partial \boldsymbol{\eta}} \cdot \delta \boldsymbol{\eta}-\frac{\partial \mathcal{W}}{\partial \mathbf{u}^{\mathrm{c}}} \cdot \delta \mathbf{u}^{\mathrm{c}}\right] d s d t=0 .
\end{aligned}
$$

For a field variable, say $\chi(s, t)$, we consider a smooth one-parameter family of curves $\chi_{\varepsilon}(s, t)$ such that $\chi_{0}(s, t)=\chi(s, t)$, and we denote by $\delta \chi$ the derivative of $\chi_{\varepsilon}$ with respect to the parameter $\varepsilon$ evaluated at $\varepsilon=0$, i.e., $\delta \chi=\left.\frac{d}{d \varepsilon} \chi_{\varepsilon}\right|_{\varepsilon=0}$.

From the orthogonality condition $\boldsymbol{R} \boldsymbol{R}^{T}=\boldsymbol{I}$, it follows that $\delta \boldsymbol{R} \boldsymbol{R}^{T}$ should be a skew-symmetric tensor. Therefore, there exists a vector $\delta \boldsymbol{q}$ such that

$$
(\delta \boldsymbol{q})^{\times}=\delta \boldsymbol{R} \boldsymbol{R}^{T}
$$

Use of (5.15) along with an interchange of mixed $(\varepsilon, s)$ and $(\varepsilon, t)$ derivatives implies that we can express variations of the variables in (5.11) in terms of the variations $\delta \boldsymbol{r}, \delta \boldsymbol{q}, \delta \boldsymbol{w}$ and $\delta \boldsymbol{\eta}$ of the independent field variables

$$
\begin{array}{ll}
\delta \mathbf{w}=\boldsymbol{R}^{T}[\delta \boldsymbol{w}+\boldsymbol{w} \times \delta \boldsymbol{q}], & \delta \boldsymbol{\eta}=\boldsymbol{R}^{T}[\delta \boldsymbol{\eta}+\boldsymbol{\eta} \times \delta \boldsymbol{q}], \\
\delta \mathbf{v}=\boldsymbol{R}^{T}\left[\delta \boldsymbol{r}_{s}+\boldsymbol{r}_{s} \times \delta \boldsymbol{q}\right], & \delta \mathbf{v}^{\mathrm{c}}=\left(\boldsymbol{R}^{T} \delta \boldsymbol{w}\right)_{s}+\left(\boldsymbol{R}^{T}[\boldsymbol{w} \times \delta \boldsymbol{q}]\right)_{s}, \\
\delta \boldsymbol{\gamma}=\boldsymbol{R}^{T}\left[\delta \boldsymbol{r}_{t}+\boldsymbol{r}_{t} \times \delta \boldsymbol{q}\right], & \delta \boldsymbol{\gamma}^{\mathrm{c}}=\left(\boldsymbol{R}^{T} \delta \boldsymbol{w}\right)_{t}+\left(\boldsymbol{R}^{T}[\boldsymbol{w} \times \delta \boldsymbol{q}]\right)_{t}, \\
\delta \mathbf{u}=\boldsymbol{R}^{T} \delta \boldsymbol{q}_{s}, & \delta \mathbf{u}^{\mathrm{c}}=\alpha\left\{\left[\boldsymbol{R}^{T}(\delta \boldsymbol{\eta}+\boldsymbol{\eta} \times \delta \boldsymbol{q})\right]_{s}-(\boldsymbol{\eta} \cdot \delta \boldsymbol{\eta}) \boldsymbol{R}^{T} \boldsymbol{u}^{\mathrm{c}}\right\}, \\
\delta \boldsymbol{\omega}=\boldsymbol{R}^{T} \delta \boldsymbol{q}_{t}, & \delta \boldsymbol{\omega}^{\mathrm{c}}=\alpha\left\{\left[\boldsymbol{R}^{T}(\delta \boldsymbol{\eta}+\boldsymbol{\eta} \times \delta \boldsymbol{q})\right]_{t}-(\boldsymbol{\eta} \cdot \delta \boldsymbol{\eta}) \boldsymbol{R}^{T} \boldsymbol{\omega}^{\mathrm{c}}\right\} .
\end{array}
$$

For smooth variations $\delta \boldsymbol{r}, \delta \boldsymbol{q}, \delta \boldsymbol{w}$ and $\delta \boldsymbol{\eta}$ that vanish at the boundary $\partial \mathscr{D}$ of the domain

$$
\mathscr{D}:=\left\{(s, t), s_{1} \leqq s \leqq s_{2}, t_{1} \leqq t \leqq t_{2}\right\}
$$


the condition (5.14), expressing the vanishing of the first variation of the action $\mathcal{A}$, becomes after some integrations by parts

$$
\begin{aligned}
\int_{t_{1}}^{t_{2}} \int_{s_{1}}^{s_{2}}\{ & {\left[-\left(\boldsymbol{R} \frac{\partial \mathcal{K}}{\partial \boldsymbol{\gamma}}\right)_{t}+\left(\boldsymbol{R} \frac{\partial \mathcal{W}}{\partial \mathbf{v}}\right)_{s}\right] \cdot \delta \boldsymbol{r} } \\
+ & \boldsymbol{R}\left[\frac{\partial \mathcal{K}}{\partial \mathbf{w}}-\left(\frac{\partial \mathcal{K}}{\partial \boldsymbol{\gamma}^{\mathrm{c}}}\right)_{t}-\frac{\partial \mathcal{W}}{\partial \mathbf{w}}+\left(\frac{\partial \mathcal{W}}{\partial \mathbf{v}^{\mathrm{c}}}\right)_{s}\right] \cdot \delta \boldsymbol{w} \\
+ & {\left[-\left(\boldsymbol{R} \frac{\partial \mathcal{K}}{\partial \boldsymbol{\omega}}\right)_{t}-\boldsymbol{r}_{t} \times\left(\boldsymbol{R} \frac{\partial \mathcal{K}}{\partial \boldsymbol{\gamma}}\right)-\boldsymbol{w} \times \boldsymbol{R}\left(\frac{\partial \mathcal{K}}{\partial \mathbf{w}}-\left(\frac{\partial \mathcal{K}}{\partial \boldsymbol{\gamma}^{\mathrm{c}}}\right)_{t}\right)\right.} \\
& -\boldsymbol{\eta} \times \boldsymbol{R}\left(\frac{\partial \mathcal{K}}{\partial \boldsymbol{\eta}}-\left(\alpha \frac{\partial \mathcal{K}}{\partial \boldsymbol{\omega}}\right)_{t}\right)+\left(\boldsymbol{R} \frac{\partial \mathcal{W}}{\partial \mathbf{u}}\right)_{s}+\boldsymbol{r}_{s} \times\left(\boldsymbol{R} \frac{\partial \mathcal{W}}{\partial \mathbf{v}}\right) \\
+ & \left.\boldsymbol{w} \times \boldsymbol{R}\left(\frac{\partial \mathcal{W}}{\partial \mathbf{w}}-\left(\frac{\partial \mathcal{W}}{\partial \mathbf{v}^{\mathrm{c}}}\right)_{s}\right)+\boldsymbol{\eta} \times \boldsymbol{R}\left(\frac{\partial \mathcal{W}}{\partial \boldsymbol{\eta}}-\left(\alpha \frac{\partial \mathcal{W}}{\partial \mathbf{u}^{\mathrm{c}}}\right)_{s}\right)\right] \cdot \delta \boldsymbol{q} \\
+ & {\left[\boldsymbol{R}\left(\frac{\partial \mathcal{K}}{\partial \boldsymbol{\eta}}-\left(\alpha \frac{\partial \mathcal{K}}{\partial \boldsymbol{\omega}^{\mathrm{c}}}\right)_{t}-\frac{\partial \mathcal{W}}{\partial \boldsymbol{\eta}}+\left(\alpha \frac{\partial \mathcal{W}}{\partial \mathbf{u}^{\mathrm{c}}}\right)_{s}\right)\right.} \\
& \left.\left.-\alpha\left(\boldsymbol{\omega}^{\mathrm{c}} \cdot \boldsymbol{R} \frac{\partial \mathcal{K}}{\partial \boldsymbol{\omega}^{\mathrm{c}}}-\boldsymbol{u}^{\mathrm{c}} \cdot \boldsymbol{R} \frac{\partial \mathcal{W}}{\partial \mathbf{u}^{\mathrm{c}}}\right) \boldsymbol{\eta}\right] \cdot \delta \boldsymbol{\eta}\right\} d s d t=0 .
\end{aligned}
$$

Because $\mathcal{L}$ is written in terms of the "quasi-velocities" $\mathbf{u}$ and $\boldsymbol{\omega}$ [3] and not in terms of the "velocities" $\boldsymbol{R}_{s}$ and $\boldsymbol{R}_{t}$ as in $\widetilde{\mathfrak{L}}$, the Euler-Lagrange equations are called Euler-Poincaré equations [18, p. 9, and Chap. 13 for a detailed historical account], or simply Poincaré equations as in [3, p. 13].

From (5.16) it follows that the Euler-Poincaré equations of the action $\mathcal{A}$ are

$$
\begin{aligned}
& \left(\boldsymbol{R} \frac{\partial \mathcal{W}}{\partial \mathbf{v}}\right)_{s}-\left(\boldsymbol{R} \frac{\partial \mathcal{K}}{\partial \boldsymbol{\gamma}}\right)_{t}=\mathbf{0} \\
& \left(\boldsymbol{R} \frac{\partial \mathcal{W}}{\partial \mathbf{u}}\right)_{s}+\boldsymbol{r}_{s} \times \boldsymbol{R} \frac{\partial \mathcal{W}}{\partial \mathbf{v}}-\left(\boldsymbol{R} \frac{\partial \mathcal{K}}{\partial \boldsymbol{\omega}}\right)_{t}-\boldsymbol{r}_{t} \times \boldsymbol{R} \frac{\partial \mathcal{K}}{\partial \boldsymbol{\gamma}} \\
& +\boldsymbol{w} \times \boldsymbol{R}\left[\frac{\partial \mathcal{W}}{\partial \mathbf{w}}-\left(\frac{\partial \mathcal{W}}{\partial \mathbf{v}^{\mathrm{c}}}\right)_{s}-\frac{\partial \mathcal{K}}{\partial \mathbf{w}}+\left(\frac{\partial \mathcal{K}}{\partial \boldsymbol{\gamma}^{\mathrm{c}}}\right)_{t}\right] \\
& +\boldsymbol{\eta} \times \boldsymbol{R}\left[\frac{\partial \mathcal{W}}{\partial \boldsymbol{\eta}}-\left(\alpha \frac{\partial \mathcal{W}}{\partial \mathbf{u}^{\mathrm{c}}}\right)_{s}-\frac{\partial \mathcal{K}}{\partial \boldsymbol{\eta}}+\left(\alpha \frac{\partial \mathcal{K}}{\partial \boldsymbol{\omega}^{\mathrm{c}}}\right)_{t}\right]=\mathbf{0}, \\
& \boldsymbol{R}\left[\frac{\partial \mathcal{W}}{\partial \mathbf{w}}-\left(\frac{\partial \mathcal{W}}{\partial \mathbf{v}^{\mathrm{c}}}\right)_{s}-\frac{\partial \mathcal{K}}{\partial \mathbf{w}}+\left(\frac{\partial \mathcal{K}}{\partial \boldsymbol{\gamma}^{\mathrm{c}}}\right)_{t}\right]=\mathbf{0}, \\
& \boldsymbol{R}\left[\frac{\partial \mathcal{W}}{\partial \boldsymbol{\eta}}-\left(\alpha \frac{\partial \mathcal{W}}{\partial \mathbf{u}^{\mathrm{c}}}\right)_{s}-\frac{\partial \mathcal{K}}{\partial \boldsymbol{\eta}}+\left(\alpha \frac{\partial \mathcal{K}}{\partial \boldsymbol{\omega}^{\mathrm{c}}}\right)_{t}\right] \\
& +\alpha\left(\omega^{\mathrm{c}} \cdot \boldsymbol{R} \frac{\partial \mathcal{K}}{\partial \boldsymbol{\omega}^{\mathrm{c}}}-\boldsymbol{u}^{\mathrm{c}} \cdot \boldsymbol{R} \frac{\partial \mathcal{W}}{\partial \mathbf{u}^{\mathrm{c}}}\right) \boldsymbol{\eta}=\mathbf{0} .
\end{aligned}
$$

If we define the functional $\overline{\mathcal{K}}$ by

$$
\overline{\mathcal{K}}\left(s ; \boldsymbol{\gamma}, \boldsymbol{\omega}, \mathbf{w}, \mathbf{w}_{t}, \boldsymbol{\eta}, \boldsymbol{\eta}_{t}\right):=\mathcal{K}\left(s ; \boldsymbol{\gamma}, \boldsymbol{\omega}, \mathbf{w}, \boldsymbol{\gamma}^{\mathrm{c}}, \boldsymbol{\eta}, \boldsymbol{\omega}^{\mathrm{c}}\right),
$$


and the functional $\overline{\mathcal{W}}$ by

$$
\overline{\mathcal{W}}\left(s ; \mathbf{v}, \mathbf{u}, \mathbf{w}, \mathbf{w}_{s}, \boldsymbol{\eta}, \mathbf{\eta}_{s}\right):=\mathcal{W}\left(s ; \mathbf{v}, \mathbf{u}, \mathbf{w}, \mathbf{v}^{\mathrm{c}}, \boldsymbol{\eta}, \mathbf{u}^{\mathrm{c}}\right),
$$

then equation (5.17c) is equivalent to $\frac{\partial \overline{\mathcal{W}}}{\partial \mathbf{w}}-\left(\frac{\partial \overline{\mathcal{W}}}{\partial \mathbf{w}_{s}}\right)_{s}=\frac{\partial \overline{\mathcal{K}}}{\partial \mathbf{w}}-\left(\frac{\partial \overline{\mathcal{K}}}{\partial \mathbf{w}_{t}}\right)_{t}$ which is similar in form to the equations obtained in strain-gradient elastic theories (also known as theories of second grade materials) [31]. However, here the displacement $\mathbf{w}$ plays the role of a strain, and the derivative $\mathbf{w}_{s}$ of the displacement plays the role of a strain gradient. In strain-gradient theories, by Euclidean invariance, the action density can not depend on the displacement, whereas here the action density can and does depend on $\mathbf{w}$ while respecting Euclidean invariance. Analogous remarks are true for $(5.17 \mathrm{~d})$ which is equivalent to $\frac{\partial \overline{\mathcal{W}}}{\partial \boldsymbol{\eta}}-\left(\frac{\partial \overline{\mathcal{W}}}{\partial \boldsymbol{\eta}_{s}}\right)_{s}=\frac{\partial \overline{\mathcal{K}}}{\partial \boldsymbol{\eta}}-\left(\frac{\partial \overline{\mathcal{K}}}{\partial \boldsymbol{\eta}_{t}}\right)_{t}$.

The condition (5.15) of vanishing of the first variation of the action yields the Euler-Poincaré equations (5.17) which are expressed in the fixed frame. However, if we use instead the variation $(\delta \mathbf{q})^{\times}=\boldsymbol{R}^{T} \delta \boldsymbol{R}$ as a variation of the rotation $\boldsymbol{R}$, the condition of the vanishing of the first variation of the action yields the EulerPoincare equations written in the moving frame. We note that for the action density given by (5.12), equations (5.17a) and (5.17b) are equivalent to $(5.10)_{2}$ and $(5.10)_{3}$ which express the principles of conservation of linear and angular momenta.

\subsection{Equations of motion and constitutive relations}

It is interesting to note that in (5.17b) the term containing $\boldsymbol{w}$ vanishes by $(5.17 \mathrm{c})$, and that the term containing $\boldsymbol{\eta}$ vanishes by $(5.17 \mathrm{~d})$. From these observations it follows after rearrangement that equations (5.17) are equivalent to

$$
\begin{aligned}
& \left(\boldsymbol{R} \frac{\partial \mathcal{W}}{\partial \mathbf{v}}\right)_{s}=\left(\boldsymbol{R} \frac{\partial \mathcal{K}}{\partial \boldsymbol{\gamma}}\right)_{t}, \\
& \left(\boldsymbol{R} \frac{\partial \mathcal{W}}{\partial \mathbf{u}}\right)_{s}+\boldsymbol{r}_{s} \times \boldsymbol{R} \frac{\partial \mathcal{W}}{\partial \mathbf{v}}=\left(\boldsymbol{R} \frac{\partial \mathcal{K}}{\partial \boldsymbol{\omega}}\right)_{t}-\boldsymbol{r}_{t} \times \boldsymbol{R} \frac{\partial \mathcal{K}}{\partial \boldsymbol{\gamma}} \\
& \left(\boldsymbol{R} \frac{\partial \mathcal{W}}{\partial \mathbf{v}^{\mathrm{c}}}\right)_{s}-\left[\boldsymbol{R} \frac{\partial \mathcal{W}}{\partial \mathbf{w}}+\boldsymbol{u} \times \boldsymbol{R} \frac{\partial \mathcal{W}}{\partial \mathbf{v}^{\mathrm{c}}}\right] \\
& =\left(\boldsymbol{R} \frac{\partial \mathcal{K}}{\partial \boldsymbol{\gamma}^{\mathrm{c}}}\right)_{t}-\left[\boldsymbol{R} \frac{\partial \mathcal{K}}{\partial \mathbf{w}}+\boldsymbol{\omega} \times \boldsymbol{R} \frac{\partial \mathcal{K}}{\partial \boldsymbol{\gamma}^{\mathrm{c}}}\right], \\
& \left(\boldsymbol{R} \frac{\partial \mathcal{W}}{\partial \mathbf{u}^{\mathrm{c}}}\right)_{s}-\left[\frac{1}{\alpha} \boldsymbol{R} \frac{\partial \mathcal{W}}{\partial \boldsymbol{\eta}}+\boldsymbol{u} \times \boldsymbol{R} \frac{\partial \mathcal{W}}{\partial \mathbf{u}^{\mathrm{c}}}-\boldsymbol{u}^{\mathrm{c}} \times\left(\eta \times \boldsymbol{R} \frac{\partial \mathcal{W}}{\partial \mathbf{u}^{\mathrm{c}}}\right)\right] \\
& =\left(\boldsymbol{R} \frac{\partial \mathcal{K}}{\partial \boldsymbol{\omega}^{\mathrm{c}}}\right)_{t}-\left[\frac{1}{\alpha} \boldsymbol{R} \frac{\partial \mathcal{K}}{\partial \boldsymbol{\eta}}+\boldsymbol{\omega} \times \boldsymbol{R} \frac{\partial \mathcal{K}}{\partial \boldsymbol{\omega}^{\mathrm{c}}}-\boldsymbol{\omega}^{\mathrm{c}} \times\left(\eta \times \boldsymbol{R} \frac{\partial \mathcal{K}}{\partial \boldsymbol{\omega}^{\mathrm{c}}}\right)\right] .
\end{aligned}
$$


In a formal Lagrangian way we introduce the variables

$$
\begin{array}{rlrl}
\boldsymbol{p} & =\boldsymbol{R} \frac{\partial \mathcal{K}}{\partial \boldsymbol{\gamma}}, & \boldsymbol{\pi} & =\boldsymbol{R} \frac{\partial \mathcal{K}}{\partial \boldsymbol{\omega}}, \\
\boldsymbol{p}^{\mathrm{c}} & =\boldsymbol{R} \frac{\partial \mathcal{K}}{\partial \boldsymbol{\gamma}^{\mathrm{c}}}, & \breve{\boldsymbol{\pi}}^{\mathrm{c}} & =\boldsymbol{R} \frac{\partial \mathcal{K}}{\partial \boldsymbol{\omega}^{\mathrm{c}}}, \\
\boldsymbol{n} & =\boldsymbol{R} \frac{\partial \mathcal{W}}{\partial \mathbf{v}}, & \boldsymbol{m} & \boldsymbol{R} \frac{\partial \mathcal{W}}{\partial \mathbf{u}}, \\
\boldsymbol{n}^{\mathrm{c}} & =\boldsymbol{R} \frac{\partial \mathcal{W}}{\partial \mathbf{v}^{\mathrm{c}}}, & \breve{\boldsymbol{m}}^{\mathrm{c}} & =\boldsymbol{R} \frac{\partial \mathcal{W}}{\partial \mathbf{u}^{\mathrm{c}}}, \\
2 \boldsymbol{\sigma} & =\boldsymbol{R} \frac{\partial \mathcal{K}}{\partial \mathbf{w}}+\boldsymbol{\omega} \times \boldsymbol{p}^{\mathrm{c}}, 2 \breve{\boldsymbol{\tau}} & =\frac{1}{\alpha} \boldsymbol{R} \frac{\partial \mathcal{K}}{\partial \boldsymbol{\eta}}+\boldsymbol{\omega} \times \breve{\boldsymbol{\pi}}^{\mathrm{c}}-\boldsymbol{\omega}^{\mathrm{c}} \times\left(\boldsymbol{\eta} \times \breve{\boldsymbol{\pi}}^{\mathrm{c}}\right), \\
2 \boldsymbol{f} & =\boldsymbol{R} \frac{\partial \mathcal{W}}{\partial \mathbf{w}}+\boldsymbol{u} \times \boldsymbol{n}^{\mathrm{c}}, 2 \breve{\boldsymbol{c}} & =\frac{1}{\alpha} \boldsymbol{R} \frac{\partial \mathcal{W}}{\partial \boldsymbol{\eta}}+\boldsymbol{u} \times \breve{\boldsymbol{m}}^{\mathrm{c}}-\boldsymbol{u}^{\mathrm{c}} \times\left(\boldsymbol{\eta} \times \breve{\boldsymbol{m}}^{\mathrm{c}}\right) .
\end{array}
$$

In fact, equations (5.19a) and (5.19b) can serve as definitions for the macroscopic and microscopic densities of linear and angular momenta, while equations $(5.19 \mathrm{c})$ and $(5.19 \mathrm{~d})$ are hyperelastic constitutive relations for the macroscopic and microscopic forces and moments. The variables $\sigma, \boldsymbol{f}, \breve{\boldsymbol{\tau}}$ and $\breve{\boldsymbol{c}}$ are defined in $(5.19 \mathrm{e}, \mathrm{f})$ such that the equations of motion (5.18) assume the form

$$
\begin{aligned}
\boldsymbol{n}_{s} & =\boldsymbol{p}_{t}, \\
\boldsymbol{m}_{s}+\boldsymbol{r}_{s} \times \boldsymbol{n} & =\boldsymbol{\pi}_{t}+\boldsymbol{r}_{t} \times \boldsymbol{p}, \\
\boldsymbol{n}_{s}^{\mathrm{c}}-2 \boldsymbol{f} & =\boldsymbol{p}_{t}^{\mathrm{c}}-2 \boldsymbol{\sigma}, \\
\breve{\boldsymbol{m}}_{s}^{\mathrm{c}}-2 \breve{\boldsymbol{c}} & =\breve{\boldsymbol{\pi}}_{t}^{\mathrm{c}}-2 \breve{\boldsymbol{\tau}} .
\end{aligned}
$$

Equations (5.20) are the equations of motion for a special Cosserat rod endowed with two microstructure vectors: a simple vector $\boldsymbol{w}$ and a Gibbs rotation vector $\boldsymbol{\eta}$. Equations $(5.20 \mathrm{a}, \mathrm{b})$ will be called the equations of macromotion, and equations $(5.20 \mathrm{c}, \mathrm{d})$ will be called the equations of micromotion.

Now we specialize to the case where the rod with the microstructures $\boldsymbol{w}$ and $\boldsymbol{\eta}$ is a birod that is composed of two special Cosserat rods bound together elastically. In such a case, it is natural to assume that the dependence of $\mathcal{W}$ on $\mathbf{u}, \mathbf{v}, \mathbf{w}_{s}$ and $\boldsymbol{\eta}_{s}$ enters only through its dependence on $\mathbf{u}^{ \pm}$and $\mathbf{v}^{ \pm}$, and the dependence of $\mathcal{K}$ on $\boldsymbol{\omega}$, $\boldsymbol{\gamma}, \mathbf{v}^{\mathfrak{c}}$ and $\mathbf{u}^{\mathrm{c}}$ enters only through its dependence on $\boldsymbol{\omega}^{ \pm}$and $\boldsymbol{\gamma}^{ \pm}$, where

$$
\begin{array}{ll}
\mathbf{v}^{ \pm}=\boldsymbol{R}^{T} \boldsymbol{P}^{ \pm T} \boldsymbol{v}^{ \pm}, & \mathbf{u}^{ \pm}=\boldsymbol{R}^{T} \boldsymbol{P}^{ \pm T} \boldsymbol{u}^{ \pm} \\
\boldsymbol{\gamma}^{ \pm}=\boldsymbol{R}^{T} \boldsymbol{P}^{ \pm T} \boldsymbol{\gamma}^{ \pm}, & \boldsymbol{\omega}^{ \pm}=\boldsymbol{R}^{T} \boldsymbol{P}^{ \pm T} \boldsymbol{\omega}^{ \pm}
\end{array}
$$

We here note that the components in the fixed frame $\left\{\boldsymbol{e}_{i}\right\}$ of the vectors $\mathbf{v}^{ \pm}, \mathbf{u}^{ \pm}$, $\boldsymbol{\gamma}^{ \pm}, \boldsymbol{\omega}^{ \pm}$coincide with the components in the moving frame $\left\{\boldsymbol{d}_{i}^{ \pm}\right\}$of the vectors $\boldsymbol{v}^{ \pm}$ $\boldsymbol{u}^{ \pm}, \boldsymbol{\gamma}^{ \pm}, \boldsymbol{\omega}^{ \pm}$, respectively.

For the sake of simplicity, in the following development we will consider the case of a birod with equal mass densities, i.e., we take $\lambda^{ \pm} \equiv 1$ in (2.4). In such a 
case, from the kinematic relations $\left(2.20^{ \pm}\right)$and $\left(2.49^{ \pm}\right)$, it follows that

$$
\begin{aligned}
& \mathbf{v}^{ \pm}=\frac{1}{g^{ \pm}} \mathbf{P}^{ \pm T}\left[\mathbf{v} \pm \mathbf{v}^{\mathrm{c}} \pm \mathbf{u} \times \mathbf{w}\right], \\
& \mathbf{u}^{ \pm}=\frac{1}{g^{ \pm}} \mathbf{P}^{ \pm T}\left[\mathbf{u} \pm \mathbf{A}^{ \pm} \mathbf{u}^{\mathrm{c}}\right] .
\end{aligned}
$$

Similarly, from the kinematic relations $\left(2.21^{ \pm}\right)$and $\left(2.50^{ \pm}\right)$we find that

$$
\begin{aligned}
\boldsymbol{\gamma}^{ \pm} & =\mathbf{P}^{ \pm T}\left[\boldsymbol{\gamma} \pm \boldsymbol{\gamma}^{\mathrm{c}} \pm \boldsymbol{\omega} \times \mathbf{w}\right], \\
\boldsymbol{\omega}^{ \pm} & =\mathbf{P}^{ \pm T}\left[\boldsymbol{\omega} \pm \mathbf{A}^{ \pm} \boldsymbol{\omega}^{\mathrm{c}}\right],
\end{aligned}
$$

where $\mathbf{P}=\boldsymbol{R}^{T} \boldsymbol{P} \boldsymbol{R}$ and $\mathbf{A}=\boldsymbol{R}^{T} \boldsymbol{A} \boldsymbol{R}$.

Now let $\widetilde{\mathcal{W}}$ be the functional defined by

$$
\tilde{\mathcal{W}}\left(s ; \mathbf{v}^{+}, \mathbf{v}^{-}, \mathbf{u}^{+}, \mathbf{u}^{-}, \mathbf{w}, \boldsymbol{\eta}\right):=\mathcal{W}\left(s ; \mathbf{v}, \mathbf{u}, \mathbf{w}, \mathbf{v}^{\mathrm{c}}, \boldsymbol{\eta}, \mathbf{u}^{\mathrm{c}}\right),
$$

and let $\widetilde{\mathcal{K}}$ be the functional defined by

$$
\widetilde{\mathcal{K}}\left(s ; \boldsymbol{\gamma}^{+}, \boldsymbol{\gamma}^{-}, \boldsymbol{\omega}^{+}, \boldsymbol{\omega}^{-}, \mathbf{w}, \boldsymbol{\eta}\right):=\mathcal{K}\left(s ; \boldsymbol{\gamma}, \boldsymbol{\omega}, \mathbf{w}, \boldsymbol{\gamma}^{\mathrm{c}}, \boldsymbol{\eta}, \boldsymbol{\omega}^{\mathrm{c}}\right) .
$$

Then, after the use of the chain rule for differentiation of composite functions, along with the relations $\left(5.21^{ \pm}\right),\left(5.22^{ \pm}\right),\left(5.23^{ \pm}\right)$and $\left(5.24^{ \pm}\right)$, the constitutive relations (5.19) become (see Appendix B)

$$
\begin{array}{rlrl}
\boldsymbol{p} & =\boldsymbol{p}^{+}+\boldsymbol{p}^{-}, & \boldsymbol{\pi}=\boldsymbol{\pi}^{+}+\boldsymbol{\pi}^{-}+\boldsymbol{w} \times\left(\boldsymbol{p}^{+}-\boldsymbol{p}^{-}\right), \\
\boldsymbol{p}^{\mathrm{c}}=\boldsymbol{p}^{+}-\boldsymbol{p}^{-}, & \boldsymbol{\pi}^{\mathrm{c}}=\boldsymbol{\pi}^{+}-\boldsymbol{\pi}^{-}+\boldsymbol{w} \times\left(\boldsymbol{p}^{+}+\boldsymbol{p}^{-}\right), \\
\boldsymbol{n} & =\boldsymbol{n}^{+}+\boldsymbol{n}^{-}, \quad & \boldsymbol{m}=\boldsymbol{m}^{+}+\boldsymbol{m}^{-}+\boldsymbol{w} \times\left(\boldsymbol{n}^{+}-\boldsymbol{n}^{-}\right), \\
\boldsymbol{n}^{\mathrm{c}} & =\boldsymbol{n}^{+}-\boldsymbol{n}^{-}, & \boldsymbol{m}^{\mathrm{c}}=\boldsymbol{m}^{+}-\boldsymbol{m}^{-}+\boldsymbol{w} \times\left(\boldsymbol{n}^{+}+\boldsymbol{n}^{-}\right), \\
2 \boldsymbol{\sigma} & =\boldsymbol{R} \frac{\partial \widetilde{\mathcal{K}}}{\partial \mathbf{w}}, \quad 2 \boldsymbol{\tau}=\frac{1}{\alpha} \boldsymbol{R} \frac{\partial \tilde{\mathcal{K}}}{\partial \boldsymbol{\eta}}-\boldsymbol{\eta} \times(\boldsymbol{w} \times 2 \boldsymbol{\sigma}), \\
2 \boldsymbol{f} & =\boldsymbol{R} \frac{\partial \tilde{\mathcal{W}}}{\partial \mathbf{w}}, \quad 2 \boldsymbol{c}=\frac{1}{\alpha} \boldsymbol{R} \frac{\partial \tilde{\mathcal{W}}}{\partial \boldsymbol{\eta}}-\boldsymbol{\eta} \times(\boldsymbol{w} \times 2 \boldsymbol{f}),
\end{array}
$$

where we have defined

$$
\begin{aligned}
& \boldsymbol{p}^{ \pm}=\boldsymbol{P}^{ \pm} \boldsymbol{R} \frac{\partial \widetilde{\mathcal{K}}}{\partial \boldsymbol{\gamma}^{ \pm}}, \quad \boldsymbol{\pi}^{ \pm}=\boldsymbol{P}^{ \pm} \boldsymbol{R} \frac{\partial \widetilde{\mathcal{K}}}{\partial \boldsymbol{\omega}^{ \pm}}, \\
& \boldsymbol{n}^{ \pm}=\frac{1}{g^{ \pm}} \boldsymbol{P}^{ \pm} \boldsymbol{R} \frac{\partial \widetilde{\mathcal{W}}}{\partial \mathbf{v}^{ \pm}}, \quad \boldsymbol{m}^{ \pm}=\frac{1}{g^{ \pm}} \boldsymbol{P}^{ \pm} \boldsymbol{R} \frac{\partial \widetilde{\mathcal{W}}}{\partial \mathbf{u}^{ \pm}},
\end{aligned}
$$

which represent the densities of linear and angular momenta, and the internal forces and moments of the individual strands.

Note that in the constitutive equations (5.27) we have used $\pi^{\mathrm{c}}, \boldsymbol{m}^{\mathrm{c}}, \boldsymbol{\tau}$ and $\boldsymbol{c}$ defined by

$$
\begin{aligned}
\pi^{\mathrm{c}} & =\breve{\boldsymbol{\pi}}^{\mathrm{c}}+\boldsymbol{w} \times \boldsymbol{p}+\boldsymbol{\eta} \times\left(\boldsymbol{\pi}-\boldsymbol{w} \times \boldsymbol{p}^{\mathrm{c}}\right), \\
\boldsymbol{m}^{\mathrm{c}} & =\breve{\boldsymbol{m}}^{\mathrm{c}}+\boldsymbol{w} \times \boldsymbol{n}+\boldsymbol{\eta} \times\left(\boldsymbol{m}-\boldsymbol{w} \times \boldsymbol{n}^{\mathrm{c}}\right), \\
2 \boldsymbol{\tau} & =2 \breve{\boldsymbol{\tau}}+\boldsymbol{w}_{t} \times \boldsymbol{p}+\boldsymbol{r}_{t} \times \boldsymbol{p}^{\mathrm{c}}+\left[\boldsymbol{\eta} \times\left(\boldsymbol{\pi}-\boldsymbol{w} \times \boldsymbol{p}^{\mathrm{c}}\right)\right]_{t}, \\
2 \boldsymbol{c} & =2 \breve{\boldsymbol{c}}+\boldsymbol{w}_{s} \times \boldsymbol{n}+\boldsymbol{r}_{s} \times \boldsymbol{n}^{\mathrm{c}}+\left[\boldsymbol{\eta} \times\left(\boldsymbol{m}-\boldsymbol{w} \times \boldsymbol{n}^{\mathrm{c}}\right)\right]_{S},
\end{aligned}
$$


in lieu of $\breve{\boldsymbol{\pi}}^{\mathrm{c}}, \breve{\boldsymbol{m}}^{\mathrm{c}}, \breve{\boldsymbol{\tau}}$ and $\breve{\boldsymbol{c}}$ used in the constitutive equations (5.19). The quantities

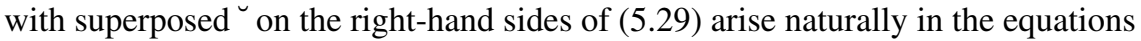
of micromoment of a rod with rather general microstructure, while the quantities on the left-hand sides of (5.29) are more appropriate for the particular case of a birod composed of two strands, because they have a direct physical interpretation.

The equations of motion (5.20) then become

$$
\begin{aligned}
\boldsymbol{n}_{s} & =\boldsymbol{p}_{t}, \\
\boldsymbol{m}_{s}+\boldsymbol{r}_{s} \times \boldsymbol{n} & =\boldsymbol{\pi}_{t}+\boldsymbol{r}_{t} \times \boldsymbol{p}, \\
\boldsymbol{n}_{s}^{\mathrm{c}}-2 \boldsymbol{f} & =\boldsymbol{p}_{t}^{\mathrm{c}}-2 \boldsymbol{\sigma}, \\
\boldsymbol{m}_{s}^{\mathrm{c}}+\boldsymbol{r}_{s} \times \boldsymbol{n}^{\mathrm{c}}-2 \boldsymbol{c} & =\boldsymbol{\pi}_{t}^{\mathrm{c}}+\boldsymbol{r}_{t} \times \boldsymbol{p}^{\mathrm{c}}-2 \boldsymbol{\tau} .
\end{aligned}
$$

When all quantities in the above equations are independent of the time $t$, the righthand sides all vanish and the equilibrium equations for the birod arise. We remark that in the constitutive equations (5.27), and in the equations of motion (5.30), we have the following exchange symmetry

$$
\begin{gathered}
s \leftrightarrow t, \quad \mathcal{W} \leftrightarrow \mathcal{K}, \quad \boldsymbol{v} \leftrightarrow \boldsymbol{\gamma}, \quad \boldsymbol{u} \leftrightarrow \boldsymbol{\omega}, \quad \boldsymbol{\eta}^{\nabla} \leftrightarrow \stackrel{\circ}{\eta}, \\
\boldsymbol{n} \leftrightarrow \boldsymbol{p}, \quad \boldsymbol{m} \leftrightarrow \boldsymbol{\pi}, \quad \boldsymbol{n}^{\mathrm{c}} \leftrightarrow \boldsymbol{p}^{\mathrm{c}}, \quad \boldsymbol{m}^{\mathrm{c}} \leftrightarrow \boldsymbol{\pi}^{\mathrm{c}}, \quad \boldsymbol{f} \leftrightarrow \boldsymbol{\sigma}, \quad \boldsymbol{c} \leftrightarrow \boldsymbol{\tau} .
\end{gathered}
$$

Comparison of the equations of motion (5.30) derived from Hamilton's principle with the equations of motion (3.11) obtained in Section 3 when $\lambda^{ \pm} \equiv 1$ reveals that $(5.30 \mathrm{~b}-\mathrm{d})$ differ from $(3.11 \mathrm{~b}-\mathrm{d})$ by the final additional terms appearing in the right-hand sides. To resolve these apparent discrepancies we need to specialize the assumed form of the kinetic energy (5.26) even further. It is sufficient to consider the kinetic energy (5.26) to be of the separated form

$$
\widetilde{\mathcal{K}}\left(s ; \boldsymbol{\gamma}^{+}, \boldsymbol{\gamma}^{-}, \boldsymbol{\omega}^{+}, \boldsymbol{\omega}^{-}, \mathbf{w}, \boldsymbol{\eta}\right)=\frac{1}{2}\left[\rho^{+}(s) \boldsymbol{\gamma}^{+} \cdot \boldsymbol{\gamma}^{+}+\rho^{-}(s) \boldsymbol{\gamma}^{-} \cdot \boldsymbol{\gamma}^{-}\right]+\mathcal{J}\left(s ; \boldsymbol{\omega}^{+}, \boldsymbol{\omega}^{-}\right),
$$

which is compatible with our definitions of the densities of linear and angular momenta as given in Section 3. With the assumption $\rho^{+}=\rho^{-}=\frac{1}{2} \rho$, it follows from $(5.27 \mathrm{a})_{1}$ and (5.28a) 1 applied to the special case (5.32), that $\boldsymbol{p}=\rho \boldsymbol{r}_{t}$ and $\boldsymbol{p}^{\mathrm{c}}=\rho \boldsymbol{w}_{t}$ which coincides with (3.4), which arose from the definition of the densities of linear momenta $\left(3.1^{ \pm}\right)$of the individual strands developed in Section 3. We therefore have $\boldsymbol{r}_{t} \times \boldsymbol{p}=\mathbf{0}$, and from (5.27e $)_{1}$ and (5.27f $)_{1}$, we obtain $\boldsymbol{\sigma}=\mathbf{0}, \boldsymbol{\tau}=\mathbf{0}$. Thus, in the case of a kinetic energy of the form (5.32), the equations of motion (5.30) derived from Hamilton's principle coincide precisely with the equations of motion (3.11) obtained previously in Section 3.

\subsection{Internal constraints}

Up to now, we have tacitly assumed that the composite rod undergoes unconstrained deformations. In this section we will modify the constitutive relations given previously to account for a priori restrictions on the deformation of the birod such as inextensibility and unshearability of the constituent strands, and analogous inter-strand constraints. 

form

An internal constraint may be written as a set of scalar equations each of the

$$
\widetilde{\mathcal{C}}\left(\boldsymbol{r}, \boldsymbol{r}_{s}, \boldsymbol{R}, \boldsymbol{R}_{s}, \boldsymbol{w}, \boldsymbol{w}_{s}, \boldsymbol{\eta}, \boldsymbol{\eta}_{s}\right)=0
$$

where $\widetilde{\mathcal{C}}$ is a scalar-valued function of its arguments. Since material constraints are unaffected by superposed rigid transformations, $\widetilde{\mathcal{C}}$ must be invariant under the transformation (5.3). Using arguments similar to those exploited in Section 5.2 we see that $\widetilde{\mathcal{C}}$ must be independent of $\boldsymbol{r}$ and $\boldsymbol{R}$. Hence, the constraint (5.33) is equivalent to the reduced form

$$
\begin{aligned}
& \mathcal{C}\left(\mathbf{v}, \mathbf{u}, \mathbf{w}, \mathbf{v}^{\mathrm{c}}, \boldsymbol{\eta}, \mathbf{u}^{\mathrm{c}}\right):= \\
& \widetilde{\mathcal{C}}\left(\boldsymbol{R}^{T} \boldsymbol{r}, \boldsymbol{R}^{T} \boldsymbol{r}_{s}, \boldsymbol{R}^{T} \boldsymbol{R}, \boldsymbol{R}^{T} \boldsymbol{R}_{s}, \boldsymbol{R}^{T} \boldsymbol{w}, \boldsymbol{R}^{T} \boldsymbol{w}_{s}, \boldsymbol{R}^{T} \boldsymbol{\eta}, \boldsymbol{R}^{T} \boldsymbol{\eta}_{s}\right)=0 .
\end{aligned}
$$

The scalar constraint (5.34) defines a hypersurface in the space $\left(\mathbf{v}, \mathbf{u}, \mathbf{w}, \mathbf{v}^{\mathbf{c}}, \boldsymbol{\eta}, \mathbf{u}^{\mathrm{c}}\right)$.

Now assume that we have $K$ scalar internal constraints given by $\mathcal{C}^{k}\left(\mathbf{v}, \mathbf{u}, \mathbf{w}, \mathbf{v}^{\mathbf{c}}\right.$, $\left.\boldsymbol{\eta}, \mathbf{u}^{\mathrm{c}}\right)=0, k=1 \ldots K$. Then the constitutive relations $(5.19)_{2}$ must be replaced by

$$
\begin{aligned}
\boldsymbol{n} & =\boldsymbol{R}\left(\frac{\partial \mathcal{W}}{\partial \mathbf{v}}+\sum_{k=1}^{K} \Lambda_{\mathbf{v}}^{k} \frac{\partial \mathcal{C}^{k}}{\partial \mathbf{v}}\right), \boldsymbol{m}=\boldsymbol{R}\left(\frac{\partial \mathcal{W}}{\partial \mathbf{u}}+\sum_{k=1}^{K} \Lambda_{\mathbf{u}}^{k} \frac{\partial \mathcal{C}^{k}}{\partial \mathbf{u}}\right) \\
\boldsymbol{n}^{\mathrm{c}} & =\boldsymbol{R}\left(\frac{\partial \mathcal{W}}{\partial \mathbf{v}^{\mathrm{c}}}+\sum_{k=1}^{K} \Lambda_{\mathbf{v}^{\mathrm{c}}}^{k} \frac{\partial \mathcal{C}^{k}}{\partial \mathbf{v}^{\mathrm{c}}}\right), \breve{\boldsymbol{m}}^{\mathrm{c}}=\boldsymbol{R}\left(\frac{\partial \mathcal{W}}{\partial \mathbf{u}^{\mathrm{c}}}+\sum_{k=1}^{K} \Lambda_{\mathbf{u}^{\mathrm{c}}}^{k} \frac{\partial \mathcal{C}^{k}}{\partial \mathbf{u}^{\mathrm{c}}}\right), \\
\boldsymbol{f} & =\boldsymbol{R}\left(\frac{\partial \mathcal{W}}{\partial \mathbf{w}}+\sum_{k=1}^{K} \Lambda_{\mathbf{w}}^{k} \frac{\partial \mathcal{C}^{k}}{\partial \mathbf{w}}\right)+\boldsymbol{u} \times \boldsymbol{n}^{\mathrm{c}} \\
\breve{\boldsymbol{c}} & =\frac{1}{2 \alpha} \boldsymbol{R}\left(\frac{\partial \mathcal{W}}{\partial \boldsymbol{\eta}}+\sum_{k=1}^{K} \Lambda_{\eta}^{k} \frac{\partial \mathcal{C}^{k}}{\partial \boldsymbol{\eta}}\right)+\boldsymbol{u} \times \breve{\boldsymbol{m}}^{\mathrm{c}}-\boldsymbol{u}^{\mathrm{c}} \times\left(\boldsymbol{\eta} \times \breve{\boldsymbol{m}}^{\mathrm{c}}\right),
\end{aligned}
$$

where $\Lambda_{\mathbf{v}}^{k}, \Lambda_{\mathbf{u}}^{k}, \Lambda_{\mathbf{v}^{\mathbf{c}}}^{k}, \Lambda_{\mathbf{u}^{\mathrm{c}}}^{k}, \Lambda_{\mathbf{w}}^{k}$ and $\Lambda_{\boldsymbol{\eta}}^{k}$ are arbitrary Lagrange multipliers called reactive parameters. These multipliers are not derivable from material properties and must be determined from the balance laws such that the constraints are satisfied.

In the present theory of birods, it is possible to consider both constraints on the individual strands such as inextensibility and unshearability, and inter-strand constraints. The constraints $\boldsymbol{v}^{ \pm} \cdot \boldsymbol{d}_{3}^{ \pm}=1$ expressing inextensibility of the strands can be written in reduced form as

$$
\mathcal{C}_{v_{3}^{ \pm}}\left(\mathbf{v}, \mathbf{u}, \mathbf{w}, \mathbf{v}^{\mathrm{c}}, \boldsymbol{\eta}, \mathbf{u}^{\mathrm{c}}\right):=\left(\mathbf{P}^{ \pm} \boldsymbol{e}_{3}\right) \cdot\left(\mathbf{v} \pm \mathbf{v}^{\mathrm{c}} \pm \mathbf{u} \times \mathbf{w}\right)-g^{ \pm}=0
$$

while the constraints $\boldsymbol{v}^{ \pm} \cdot \boldsymbol{d}_{i}^{ \pm}=0, i=1,2$, expressing unshearability of the strands can be written in reduced form as

$$
\mathcal{C}_{v_{i}^{ \pm}}\left(\mathbf{v}, \mathbf{u}, \mathbf{w}, \mathbf{v}^{\mathrm{c}}, \boldsymbol{\eta}, \mathbf{u}^{\mathrm{c}}\right):=\left(\mathbf{P}^{ \pm} \boldsymbol{e}_{i}\right) \cdot\left(\mathbf{v} \pm \mathbf{v}^{\mathrm{c}} \pm \mathbf{u} \times \mathbf{w}\right)=0, \quad i=1,2
$$


Here we recall that

$$
\mathbf{P}^{ \pm}=\boldsymbol{I} \pm \frac{2}{1+\|\boldsymbol{\eta}\|^{2}}\left[\boldsymbol{I} \pm \boldsymbol{\eta}^{\times}\right] \boldsymbol{\eta}^{\times}
$$

As for restrictions on the inter-strand deformation we can impose constraints of the type $\boldsymbol{w} \cdot \boldsymbol{d}_{i}=\hat{w}_{i}, i=1,2,3$, which restrict inter-strand translational deformation, and which can be written in the form (5.34) with

$$
\mathcal{C}_{w_{i}}\left(\mathbf{v}, \mathbf{u}, \mathbf{w}, \mathbf{v}^{\mathrm{c}}, \boldsymbol{\eta}, \mathbf{u}^{\mathrm{c}}\right):=\mathbf{w} \cdot \boldsymbol{e}_{i}-\hat{w}_{i}=0, \quad i=1,2,3
$$

Constraints of the type $\boldsymbol{\eta} \cdot \boldsymbol{d}_{i}=\hat{\eta}_{i}, i=1,2,3$, which restrict inter-strand rotational deformation, can be written in the form (5.34) with

$$
\mathcal{C}_{\eta_{i}}\left(\mathbf{v}, \mathbf{u}, \mathbf{w}, \mathbf{v}^{\mathrm{c}}, \boldsymbol{\eta}, \mathbf{u}^{\mathrm{c}}\right):=\boldsymbol{\eta} \cdot \boldsymbol{e}_{i}-\hat{\eta}_{i}=0, \quad i=1,2,3
$$

For a composite rod with some of the above mentioned constraints, equations (5.35) enable us to derive appropriate constitutive relations. However, in the remainder of this article we will continue to work with the unconstrained theory.

\subsection{Boundary conditions}

For smooth variations $\delta \boldsymbol{r}, \delta \boldsymbol{q}, \delta \boldsymbol{w}$ and $\delta \eta$, vanishing at $t=t_{1}$ and $t=t_{2}$, but otherwise arbitrary, the vanishing of the first variation of the action requires that in addition to (5.16) we must have

$$
\begin{aligned}
\int_{t_{1}}^{t_{2}}\left[\left(\boldsymbol{R} \frac{\partial \mathcal{W}}{\partial \mathbf{v}}\right)\right. & \cdot \delta \boldsymbol{r}+\left(\boldsymbol{R} \frac{\partial \mathcal{W}}{\partial \mathbf{v}^{\mathrm{c}}}\right) \cdot \delta \boldsymbol{w}+\left(\boldsymbol{R} \frac{\partial \mathcal{W}}{\partial \mathbf{u}}-\boldsymbol{w} \times \boldsymbol{R} \frac{\partial \mathcal{W}}{\partial \mathbf{v}^{\mathrm{c}}}\right. \\
& \left.\left.-\alpha \boldsymbol{\eta} \times \boldsymbol{R} \frac{\partial \mathcal{W}}{\partial \mathbf{u}^{\mathrm{c}}}\right) \cdot \delta \boldsymbol{q}+\left(\alpha \boldsymbol{R} \frac{\partial \mathcal{W}}{\partial \mathbf{u}^{\mathrm{c}}}\right) \cdot \delta \boldsymbol{\eta}\right]_{s=s_{1}}^{s=s_{2}} d t=0 .
\end{aligned}
$$

Therefore, in view of the constitutive relations (5.19), whenever an essential boundary condition of the form

$$
\begin{array}{lr}
\left.\boldsymbol{r}\right|_{s=\xi_{1}}=\boldsymbol{r}^{*}, & \left.\boldsymbol{q}\right|_{s=\xi_{2}}=\boldsymbol{q}^{*}, \\
\left.\boldsymbol{w}\right|_{s=\xi_{3}}=\boldsymbol{w}^{*}, & \left.\boldsymbol{\eta}\right|_{s=\xi_{4}}=\boldsymbol{\eta}^{*},
\end{array}
$$

with prescribed vectors $\boldsymbol{r}^{*}, \boldsymbol{q}^{*}, \boldsymbol{w}^{*}$ and $\boldsymbol{\eta}^{*}$, is not imposed, we obtain natural boundary conditions of the form

$$
\begin{aligned}
& \left.\boldsymbol{n}\right|_{s=\xi_{1}}=\mathbf{0},\left.\quad \boldsymbol{n}^{\mathrm{c}}\right|_{s=\xi_{3}}=\mathbf{0}, \\
& \boldsymbol{m}-\boldsymbol{w} \times \boldsymbol{n}^{\mathrm{c}}-\alpha \boldsymbol{\eta} \times\left.\left[\boldsymbol{m}^{\mathrm{c}}-\boldsymbol{w} \times \boldsymbol{n}-\boldsymbol{\eta} \times\left(\boldsymbol{m}-\boldsymbol{w} \times \boldsymbol{n}^{\mathrm{c}}\right)\right]\right|_{s=\xi_{2}}=\mathbf{0}, \\
& \boldsymbol{m}^{\mathrm{c}}-\boldsymbol{w} \times \boldsymbol{n}-\boldsymbol{\eta} \times\left.\left(\boldsymbol{m}-\boldsymbol{w} \times \boldsymbol{n}^{\mathrm{c}}\right)\right|_{s=\xi_{4}}=\mathbf{0},
\end{aligned}
$$

where $\xi_{i}, i=1, \ldots, 4$ is equal to either $s_{1}$ or $s_{2}$. 


\section{Linearly-elastic constitutive equations}

With the objective of making our general theory more concrete, in this section we further develop the theory for the particular case of decoupled, linearly-elastic constitutive relations in which the strain energy density (5.25) is taken to be of the quadratic, decoupled form

$$
\tilde{\mathcal{W}}\left(s ; \mathbf{v}^{+}, \mathbf{v}^{-}, \mathbf{u}^{+}, \mathbf{u}^{-}, \mathbf{w}, \boldsymbol{\eta}\right)=\tilde{\mathcal{W}}^{+}\left(s ; \mathbf{v}^{+}, \mathbf{u}^{+}\right)+\tilde{\mathcal{W}}^{-}\left(s ; \mathbf{v}^{-}, \mathbf{u}^{-}\right)+\tilde{\mathcal{W}}^{\mathrm{c}}(s ; \mathbf{w}, \boldsymbol{\eta}),
$$

with

$$
\begin{aligned}
\tilde{\mathcal{W}}^{ \pm}\left(s ; \mathbf{v}^{ \pm}, \mathbf{u}^{ \pm}\right) & =\frac{1}{2}\left(\mathbf{v}^{ \pm}-\check{\mathbf{v}}^{ \pm}\right) \cdot \mathbf{L}^{ \pm}\left(\mathbf{v}^{ \pm}-\check{\mathbf{v}}^{ \pm}\right)+\frac{1}{2}\left(\mathbf{u}^{ \pm}-\check{\mathbf{u}}^{ \pm}\right) \cdot \mathbf{K}^{ \pm}\left(\mathbf{u}^{ \pm}-\check{\mathbf{u}}^{ \pm}\right), \\
\tilde{\mathcal{W}}^{\mathrm{c}}(s ; \mathbf{w}, \boldsymbol{\eta}) & =\frac{1}{2}(\mathbf{w}-\check{\mathbf{w}}) \cdot \mathbf{H}(\mathbf{w}-\check{\mathbf{w}})+\frac{1}{2}(\boldsymbol{\eta}-\check{\boldsymbol{\eta}}) \cdot \mathbf{G}(\boldsymbol{\eta}-\check{\boldsymbol{\eta}}),
\end{aligned}
$$

where $\mathbf{L}^{ \pm}(s), \mathbf{K}^{ \pm}(s), \mathbf{H}(s)$, and $\mathbf{G}(s)$ are symmetric, positive-definite tensor functions of $s$ only. Here $\check{\mathbf{v}}^{ \pm}(s)$ and $\check{\mathbf{u}}^{ \pm}(s)$ denote quantities associated with the stressfree configuration of the individual strands, which may or may not correspond to the stress-free configuration of the birod. All quantities with a superposed ${ }^{`}$, such as $\check{\mathbf{w}}$, are given shape parameters.

The kinetic energy density (5.26) is taken to be of the form

$$
\widetilde{\mathcal{K}}\left(s ; \boldsymbol{\gamma}^{+}, \boldsymbol{\gamma}^{-}, \boldsymbol{\omega}^{+}, \boldsymbol{\omega}^{-}, \mathbf{w}, \boldsymbol{\eta}\right)=\frac{1}{2}\left\{\rho^{+} \boldsymbol{\gamma}^{+} \cdot \boldsymbol{\gamma}^{+}+\boldsymbol{\omega}^{+} \cdot \mathbf{J}^{+} \boldsymbol{\omega}^{+}+\rho^{-} \boldsymbol{\gamma}^{-} \cdot \boldsymbol{\gamma}^{-}+\boldsymbol{\omega}^{-} \cdot \mathbf{J}^{-} \boldsymbol{\omega}^{-}\right\},
$$

where $\mathbf{J}^{ \pm}(s)$ are symmetric, positive-definite tensor functions of $s$ only.

Using $(5.27 \mathrm{a}, \mathrm{b})$ and $(5.28 \mathrm{a})_{1}$ we find that the macroscopic and microscopic densities of linear momentum are given by

$$
\left(\begin{array}{c}
\boldsymbol{p} \\
\boldsymbol{p}^{\mathrm{c}}
\end{array}\right)=\rho\left(\begin{array}{c}
\boldsymbol{r}_{t} \\
\boldsymbol{w}_{t}
\end{array}\right),
$$

where $\rho=\rho(s)=\rho^{+}(s)+\rho^{-}(s)$. While in Section 3 we took (3.4) as the definitions for the macroscopic and microscopic densities of linear momentum, the development of Section 5 reveals that they also naturally follow from a constitutive assumption in which the kinetic energy is an isotropic quadratic form in the macroscopic and microscopic linear velocities as in (6.2). To obtain expressions for the macroscopic and microscopic densities of angular momentum, we introduce the symmetric tensors

$$
\boldsymbol{J}=\boldsymbol{J}^{+}+\boldsymbol{J}^{-}, \quad \boldsymbol{J}^{\mathrm{c}}=\boldsymbol{J}^{+}-\boldsymbol{J}^{-},
$$

where the symmetric positive-definite tensors $\boldsymbol{J}^{ \pm}$, given by

$$
\boldsymbol{J}^{ \pm}=\boldsymbol{P}^{ \pm} \boldsymbol{R} \mathbf{J}^{ \pm} \boldsymbol{R}^{T} \boldsymbol{P}^{ \pm T},
$$

represent the moment of inertia contributions, in the current configuration, of the strands $\mathscr{R}^{ \pm}$to the moment of inertia of the birod $\mathscr{R}$. Then, with (5.27) and (5.28a) we find

$$
\left(\begin{array}{c}
\pi \\
\pi^{\mathrm{c}}
\end{array}\right)=\left(\begin{array}{cc}
J & J^{\mathrm{c}} \\
J^{\mathrm{c}} & J
\end{array}\right)\left(\begin{array}{c}
\omega+\eta \times \omega^{\mathrm{c}} \\
\omega^{\mathrm{c}}
\end{array}\right)+\left(\begin{array}{c}
\boldsymbol{w} \times \boldsymbol{p}^{\mathrm{c}} \\
\boldsymbol{w} \times \boldsymbol{p}
\end{array}\right) .
$$


It is worthy to note that the coefficient matrices $\boldsymbol{R}^{T} \boldsymbol{J} \boldsymbol{R}$ and $\boldsymbol{R}^{T} \boldsymbol{J}^{\mathrm{c}} \boldsymbol{R}$ are configuration dependent, but that this dependence is only through the microrotation $\boldsymbol{\eta}$.

From (5.28b) $)_{1}$ and (6.1) it follows that the components of the internal forces of the individual strands are given by

$$
\boldsymbol{n}^{ \pm}=\frac{1}{g^{ \pm}} \boldsymbol{P}^{ \pm} \boldsymbol{R} \mathbf{L}^{ \pm}\left[\mathbf{v}^{ \pm}-\check{\mathbf{v}}^{ \pm}\right]
$$

Similarly, from (5.28b) 2 and (6.1), it follows that the components of the internal moments of the individual strands are given by

$$
\boldsymbol{m}^{ \pm}=\frac{1}{g^{ \pm}} \boldsymbol{P}^{ \pm} \boldsymbol{R} \mathbf{K}^{ \pm}\left[\mathbf{u}^{ \pm}-\check{\mathbf{u}}^{ \pm}\right] .
$$

With the constitutive equations $\left(6.6^{ \pm}\right)$for $\boldsymbol{n}^{ \pm}$we can compute expressions for $\boldsymbol{n}$ and $\boldsymbol{n}^{\mathrm{c}}$. We introduce the symmetric tensors

$$
\boldsymbol{L}=\boldsymbol{L}^{+}+\boldsymbol{L}^{-}, \quad \boldsymbol{L}^{\mathrm{c}}=\boldsymbol{L}^{+}-\boldsymbol{L}^{-},
$$

where the symmetric, positive-definite tensors $\boldsymbol{L}^{ \pm}$, defined by

$$
\boldsymbol{L}^{ \pm}=\frac{1}{\left(g^{ \pm}\right)^{2}} \boldsymbol{P}^{ \pm} \boldsymbol{R} \mathbf{L}^{ \pm} \boldsymbol{R}^{T} \boldsymbol{P}^{ \pm T}
$$

represent the stiffness (in extension and shear) contributions, in the current configuration, of the strands $\mathscr{R}^{ \pm}$to the stiffness of the birod $\mathscr{R}$. Then, with $\left(5.21^{ \pm}\right)$and $\left(6.6^{ \pm}\right)$, we conclude that $\boldsymbol{n}$ and $\boldsymbol{n}^{\mathrm{c}}$ are given by

$$
\left(\begin{array}{c}
\boldsymbol{n} \\
\boldsymbol{n}^{\mathrm{c}}
\end{array}\right)=\left(\begin{array}{cc}
\boldsymbol{L} & \boldsymbol{L}^{\mathrm{c}} \\
\boldsymbol{L}^{\mathrm{c}} & \boldsymbol{L}
\end{array}\right)\left\{\left(\begin{array}{c}
\boldsymbol{v} \\
\boldsymbol{v}^{\mathrm{c}}+\boldsymbol{w} \times \boldsymbol{u}
\end{array}\right)-\left(\begin{array}{c}
g^{+} \boldsymbol{P} \boldsymbol{R} \check{\mathbf{v}}^{+}+g^{-} \boldsymbol{P}^{T} \boldsymbol{R} \check{\mathbf{v}}^{-} \\
g^{+} \boldsymbol{P} \boldsymbol{R} \check{\mathbf{v}}^{+}-g^{-} \boldsymbol{P}^{T} \boldsymbol{R} \check{\mathbf{v}}^{-}
\end{array}\right)\right\} .
$$

We note that the effective stiffnesses for shear and extension $\boldsymbol{L}$ and $\boldsymbol{L}^{\mathrm{c}}$ again depend on the present configuration, but only through the microrotation variable $\boldsymbol{\eta}$.

Similarly, to compute the expressions for $\boldsymbol{m}$ and $\boldsymbol{m}^{\mathrm{c}}$ from the constitutive equations $\left(6.6^{ \pm}\right)$for $\boldsymbol{m}^{ \pm}$, we introduce the symmetric tensors

$$
\boldsymbol{K}=\boldsymbol{K}^{+}+\boldsymbol{K}^{-}, \quad \boldsymbol{K}^{\mathrm{c}}=\boldsymbol{K}^{+}-\boldsymbol{K}^{-},
$$

where the symmetric, positive-definite tensors $\boldsymbol{K}^{ \pm}$, given by

$$
\boldsymbol{K}^{ \pm}=\frac{1}{\left(g^{ \pm}\right)^{2}} \boldsymbol{P}^{ \pm} \boldsymbol{R} \mathbf{K}^{ \pm} \boldsymbol{R}^{T} \boldsymbol{P}^{ \pm T}
$$

represent the stiffness (in bending and twisting) contributions, in the current configuration, of the strands $\mathscr{R}^{ \pm}$to the stiffness of the birod $\mathscr{R}$. Then, with $\left(5.22^{ \pm}\right)$ and $\left(6.7^{ \pm}\right)$, we conclude that $\boldsymbol{m}$ and $\boldsymbol{m}^{\mathrm{c}}$ are given by

$$
\begin{aligned}
\left(\begin{array}{c}
\boldsymbol{m} \\
\boldsymbol{m}^{\mathrm{c}}
\end{array}\right)= & \left(\begin{array}{l}
\boldsymbol{K} \boldsymbol{K}^{\mathrm{c}} \\
\boldsymbol{K}^{\mathrm{c}} \boldsymbol{K}
\end{array}\right)\left\{\left(\begin{array}{c}
\boldsymbol{u}+\boldsymbol{\eta} \times \boldsymbol{u}^{\mathrm{c}} \\
\boldsymbol{u}^{\mathrm{c}}
\end{array}\right)-\left(\begin{array}{c}
g^{+} \boldsymbol{P} \boldsymbol{R} \check{\mathbf{u}}^{+}+g^{-} \boldsymbol{P}^{T} \boldsymbol{R} \check{\mathbf{u}}^{-} \\
g^{+} \boldsymbol{P} \boldsymbol{R} \check{\mathbf{u}}^{+}-g^{-} \boldsymbol{P}^{T} \boldsymbol{R} \check{\mathbf{u}}^{-}
\end{array}\right)\right\} \\
& +\left(\begin{array}{c}
\boldsymbol{w} \times \boldsymbol{n}^{\mathrm{c}} \\
\boldsymbol{w} \times \boldsymbol{n}
\end{array}\right) .
\end{aligned}
$$


Once again we remark that the effective stiffnesses for bending and twisting $\boldsymbol{K}$ and $\boldsymbol{K}^{\mathrm{c}}$ are configuration dependent.

Finally, from (5.27f) it follows that the components of the inter-strand force and couple are given by

$$
\begin{aligned}
2 \boldsymbol{f} & =\boldsymbol{R} \mathbf{H} \boldsymbol{R}^{T}[\boldsymbol{w}-\boldsymbol{R} \check{\mathbf{w}}], \\
2 \boldsymbol{c} & =\frac{1}{\alpha} \boldsymbol{R} \mathbf{G}^{T}[\boldsymbol{\eta}-\boldsymbol{R} \check{\boldsymbol{\eta}}]-\eta \times(\boldsymbol{w} \times 2 \boldsymbol{f}) .
\end{aligned}
$$

A few remarks are in order. First, the term $\boldsymbol{\eta} \times(\boldsymbol{w} \times 2 \boldsymbol{f})$ in $(6.12 b)$, which can be rewritten as $\frac{1}{2 \alpha}\left(\boldsymbol{P}-\boldsymbol{P}^{T}\right)(\boldsymbol{w} \times 2 \boldsymbol{f})$, is somehow counterintuitive. This term captures the interplay between rotational and translational microdisplacements. We note that this term vanishes when $f$ is parallel to $\boldsymbol{w}$, or when $\boldsymbol{\eta}$ is parallel to the moment $\boldsymbol{w} \times \boldsymbol{f}$ of the force $\boldsymbol{f}$ about $\boldsymbol{r}$. Such is the case for planar birods. Second, in the case where the elastic energy associated with the microrotation is assumed to be $\frac{1}{2}(\boldsymbol{\theta}-\check{\boldsymbol{\theta}}) \cdot \overline{\mathbf{G}}(\boldsymbol{\theta}-\check{\boldsymbol{\theta}})$, i.e., quadratic in $\boldsymbol{\theta}=\frac{2 \arctan \|\boldsymbol{\eta}\|}{\|\boldsymbol{\eta}\|} \boldsymbol{\eta}$, which is perhaps more natural than being quadratic in $\eta$ as in (6.1), then, with the chain rule, we obtain

$$
2 c=\frac{1}{\alpha} \boldsymbol{R} \mathbf{T} \overline{\mathbf{G}} \boldsymbol{R}^{T}[\boldsymbol{\theta}-\boldsymbol{R} \check{\boldsymbol{\theta}}]-\boldsymbol{\eta} \times(\boldsymbol{w} \times 2 \boldsymbol{f}),
$$

where

$$
\mathbf{T}=\frac{1}{\|\boldsymbol{\theta}\|^{2}}\left\{\alpha \boldsymbol{\theta} \otimes \boldsymbol{\theta}+\frac{\|\boldsymbol{\theta}\|}{\tan \frac{1}{2}\|\boldsymbol{\theta}\|}\left[\|\boldsymbol{\theta}\|^{2} \boldsymbol{I}-\boldsymbol{\theta} \otimes \boldsymbol{\theta}\right]\right\}
$$

\section{Boundary-value problems}

In this section we formulate and solve some very simple, illustrative, boundaryvalue problems for configurations of a birod that is made by bonding two uniform, linearly elastic strands. We assume a single common bending rigidity $K$ for both strands, and, when they are extensible, we denote by $L$ their common stretching rigidity. We assume that the strands in their intrinsic stress-free shape are arcs of circles of equal lengths, but with different curvatures (possibly zero) that we denote by $\kappa^{ \pm}$.

\subsection{Biring}

We consider a birod that is made of two linearly elastic, isotropic $\left(\kappa^{ \pm}=0\right)$ strands for which the unstressed reference configuration is described by

$$
\hat{\boldsymbol{r}}=s_{3}, \quad \hat{\boldsymbol{w}}=\hat{d} \boldsymbol{e}_{2}, \quad \hat{\boldsymbol{R}}=\boldsymbol{I}, \quad \hat{\boldsymbol{P}}=\boldsymbol{I},
$$

with $s \in[0,2 \pi R]$. There are then exactly two equilibrium configurations such that the center line $\boldsymbol{r}$ is circular: 
i) A biring with low strain energy for which the configuration is described by

$$
\boldsymbol{r}=R\left(\cos \frac{s}{R} \boldsymbol{e}_{1}+\sin \frac{s}{R} \boldsymbol{e}_{3}\right), \quad \boldsymbol{w}=\hat{d} \boldsymbol{e}_{2}, \quad \boldsymbol{P}=\boldsymbol{I},
$$

and $\boldsymbol{R}$ is a rotation through the angle $s / R$ about $\boldsymbol{e}_{2}$, while the forces and moments are given by

$$
\boldsymbol{n}=\mathbf{0}, \quad \boldsymbol{n}^{\mathrm{c}}=\mathbf{0}, \quad \boldsymbol{m}=\frac{2 K}{R} \boldsymbol{e}_{2}, \quad \boldsymbol{m}^{\mathrm{c}}=\mathbf{0} .
$$

In this case the two strands are each unstretched and are non-interacting.

ii) A biring with high strain energy for which the configuration is described by

$$
\boldsymbol{r}=R\left(\cos \frac{s}{R} \boldsymbol{e}_{2}+\sin \frac{s}{R} \boldsymbol{e}_{3}\right), \quad \boldsymbol{w}=\frac{d}{R} \boldsymbol{r}, \quad d=\frac{G \hat{d}}{L / R^{2}+G}, \quad \boldsymbol{P}=\boldsymbol{I},
$$

and $\boldsymbol{R}$ is a rotation through the angle $s / R$ about $\boldsymbol{e}_{1}$, while the forces and moments are given by

$$
\boldsymbol{n}=\mathbf{0}, \quad \boldsymbol{n}^{\mathrm{c}}=\frac{L d}{R} \boldsymbol{r}_{s}, \quad \boldsymbol{m}=\frac{2\left(K+L d^{2}\right)}{R} \boldsymbol{e}_{1}, \quad \boldsymbol{m}^{\mathrm{c}}=\mathbf{0} .
$$

Here one strand is uniformly stretched and the other is uniformly shortened. The strands interact with a transverse, radial force $2 \boldsymbol{f}=\boldsymbol{n}^{\mathrm{c}}{ }_{s}=-\frac{L d}{R^{3}} \boldsymbol{r}$.

\subsection{Stress-free configuration of a planar birod}

Given the stress-free shapes of the strands, the problem of finding the stressfree configuration of the birod is in general already a non-trivial boundary-value problem. For such a problem, we seek the position vector $\hat{\boldsymbol{r}}$, the microdisplacement vector $\hat{\boldsymbol{w}}$, the rotation tensor $\hat{\boldsymbol{R}}$, and the microrotation tensor $\hat{\boldsymbol{P}}$ of the birod that solve the equilibrium equations

$$
\begin{aligned}
\hat{\boldsymbol{n}}_{s} & =\mathbf{0}, & \hat{\boldsymbol{m}}_{s}+\hat{\boldsymbol{r}}_{s} \times \hat{\boldsymbol{n}} & =\mathbf{0}, \\
\hat{\boldsymbol{n}}_{s}^{\mathrm{c}}-2 \hat{\boldsymbol{f}} & =\mathbf{0}, & \hat{\boldsymbol{m}}_{s}^{\mathrm{c}}+\hat{\boldsymbol{r}}_{s} \times \hat{\boldsymbol{n}}^{\mathrm{c}}-2 \hat{\boldsymbol{c}} & =\mathbf{0},
\end{aligned}
$$

subject to the free or natural boundary conditions

$$
\begin{array}{cccc}
\hat{\boldsymbol{n}}\left(s_{1}\right)=\mathbf{0}, & \hat{\boldsymbol{n}}\left(s_{2}\right)=\mathbf{0}, & \hat{\boldsymbol{m}}\left(s_{1}\right)=\mathbf{0}, & \hat{\boldsymbol{m}}\left(s_{2}\right)=\mathbf{0}, \\
\hat{\boldsymbol{n}}^{\mathrm{c}}\left(s_{1}\right)=\mathbf{0}, & \hat{\boldsymbol{n}}^{\mathrm{c}}\left(s_{2}\right)=\mathbf{0}, & \hat{\boldsymbol{m}}^{\mathrm{c}}\left(s_{1}\right)=\mathbf{0}, & \hat{\boldsymbol{m}}^{\mathrm{c}}\left(s_{2}\right)=\mathbf{0} .
\end{array}
$$

Boundary conditions (7.2) are the appropriate simplification of the natural boundary conditions (5.36) where there are no imposed boundary conditions. Equations (7.1a) and boundary conditions (7.2a) integrate to imply $\hat{\boldsymbol{n}}=\mathbf{0}$ and $\hat{\boldsymbol{m}}=\mathbf{0}$. The remaining equations taken together with the appropriate constitutive relations yield a two-point boundary-value problem for the reference state.

We now formulate a specific problem for the case of a planar birod made by gluing together two inextensible strands. Let $x(s), y(s), a(s), b(s), \vartheta(s), \vartheta^{\mathrm{c}}(s)$, $\mu(s), v(s)$ be real-valued functions such that $\hat{\boldsymbol{r}}=x \boldsymbol{e}_{3}+y \boldsymbol{e}_{1}, \hat{\boldsymbol{w}}=\left(a \boldsymbol{e}_{3}+b \boldsymbol{e}_{1}\right)$, 
and $\hat{\boldsymbol{n}}^{\mathrm{c}}=K\left(\mu \boldsymbol{e}_{3}+v \boldsymbol{e}_{1}\right) . \hat{\boldsymbol{R}}$ is the rotation through the angle $\vartheta$ about $\boldsymbol{e}_{2}$, and $\hat{\boldsymbol{P}}$ is the rotation through the angle $\vartheta^{\mathrm{c}}$ about $\boldsymbol{e}_{2}$. The constitutive equations (6.11) with $\mathbf{K}^{ \pm}=K\left(\hat{\mathbf{d}}_{1}^{ \pm} \otimes \hat{\mathbf{d}}_{1}^{ \pm}+\hat{\mathbf{d}}_{2}^{ \pm} \otimes \hat{\mathbf{d}}_{2}^{ \pm}\right)+K_{3} \hat{\mathbf{d}}_{3}^{ \pm} \otimes \hat{\mathbf{d}}_{3}^{ \pm}$then yield

$$
\begin{aligned}
& \hat{\boldsymbol{m}}=K\left(\vartheta_{s}-\kappa^{+}-\kappa^{-}\right) \boldsymbol{e}_{2}+\hat{\boldsymbol{w}} \times \hat{\boldsymbol{n}}^{\mathrm{c}}, \\
& \hat{\boldsymbol{m}}^{\mathrm{c}}=K\left(\vartheta_{s}^{\mathrm{c}}-\kappa^{+}+\kappa^{-}\right) \boldsymbol{e}_{2}+\hat{\boldsymbol{w}} \times \hat{\boldsymbol{n}} .
\end{aligned}
$$

For the binding force and couple we use the constitutive relations (6.12a) with $\mathbf{H}=H \boldsymbol{I}$ and (6.13) with $\check{\boldsymbol{\theta}}=\mathbf{0}, \overline{\mathbf{G}}=G \boldsymbol{I}$, and assume that $\mathbf{T}$ is close to the identity tensor, to conclude

$$
2 \hat{\boldsymbol{f}}=H\left(\hat{\boldsymbol{w}}-\hat{\boldsymbol{R}} d \boldsymbol{e}_{2}\right), \quad 2 \hat{\boldsymbol{c}}=G \vartheta^{\mathrm{c}} \boldsymbol{e}_{2},
$$

where $G$ and $H$ are positive constants.

Exploitation of symmetry about the midpoint implies that problem (7.1)-(7.2) is equivalent to the following two-point boundary problem defined, after rescaling if necessary, on the interval $[0,1]$, and composed of the differential equations

$$
\begin{array}{rlrl}
\vartheta_{s s} & =(\mu \cos \vartheta+v \sin \vartheta) \sin \vartheta^{\mathrm{c}}+h d(a \cos \vartheta+b \sin \vartheta), \\
\vartheta_{s s}^{\mathrm{c}} & =(\mu \sin \vartheta-v \cos \vartheta) \cos \vartheta^{\mathrm{c}}+g \vartheta^{\mathrm{c}}, \\
\mu_{s} & =h(a+d \sin \vartheta), & v-s & =h(b-d \cos \vartheta), \\
a_{s} & =-\sin \vartheta \sin \vartheta^{\mathrm{c}}, & b_{s} & =\cos \vartheta \sin \vartheta^{\mathrm{c}}, \\
x_{s} & =\cos \vartheta \cos \vartheta^{\mathrm{c}}, & y_{s} & =\sin \vartheta \cos \vartheta^{\mathrm{c}},
\end{array}
$$

and the boundary conditions

$$
\begin{gathered}
\vartheta(0)=0, \vartheta^{\mathrm{c}}(0)=0, a(0)=0, x(0)=0, y(0)=0, v(0)=0, \\
\mu(1)=0, v(1)=0, \vartheta_{s}(1)=\frac{1}{2}\left(\kappa^{+}+\kappa^{-}\right), \vartheta_{s}^{\mathrm{c}}(1)=\frac{1}{2}\left(\kappa^{+}-\kappa^{-}\right),
\end{gathered}
$$

where we have set $g=G / K$ and $h=H / K$.

Before presenting numerical solutions of this boundary-value problem, we note that when $\kappa^{+}=-\kappa^{-}$there are solutions of (7.3) such that the birod is straight, i.e., $\vartheta \equiv 0$. Furthermore, for such solutions we must have $v \equiv 0, y \equiv 0$, and $a \equiv 0$, and $\vartheta^{\mathrm{c}}, \mu, b, x$ must be solutions of the reduced boundary-value problem

$$
\vartheta_{s s}^{\mathrm{c}}=-v \cos \vartheta^{\mathrm{c}}+g \vartheta^{\mathrm{c}}, v_{s}=h(b-d), b_{s}=\sin \vartheta^{\mathrm{c}}, x_{s}=\cos \vartheta^{\mathrm{c}},
$$

with boundary conditions

$$
\vartheta^{\mathrm{c}}(0)=0, \vartheta_{s}^{\mathrm{c}}(1)=\frac{1}{2}\left(\kappa^{+}-\kappa^{-}\right), v(0)=0, v(1)=0, x(0)=0 .
$$

This boundary-value problem reduces to the following fourth-order differential equation for $\vartheta^{\mathrm{c}}$,

$$
\left(\frac{\vartheta_{s s}^{c}-g \vartheta^{c}}{\cos \vartheta^{c}}\right)_{s s}+h \sin \vartheta^{c}=0,
$$

subject to the boundary conditions

$$
\vartheta^{\mathrm{c}}(0)=0, \quad \vartheta_{s s}^{\mathrm{c}}(0)=0, \quad \vartheta_{s}^{\mathrm{c}}(1)=\frac{1}{2}\left(\kappa^{+}-\kappa^{-}\right), \quad \vartheta_{s S}^{\mathrm{c}}(1)=g \vartheta^{\mathrm{c}}(1) .
$$

There is no solution of the boundary-value problem (7.3) for which $\vartheta^{\mathrm{c}} \equiv 0$ except in the trivial case of a straight birod made of intrinsically-straight strands, 

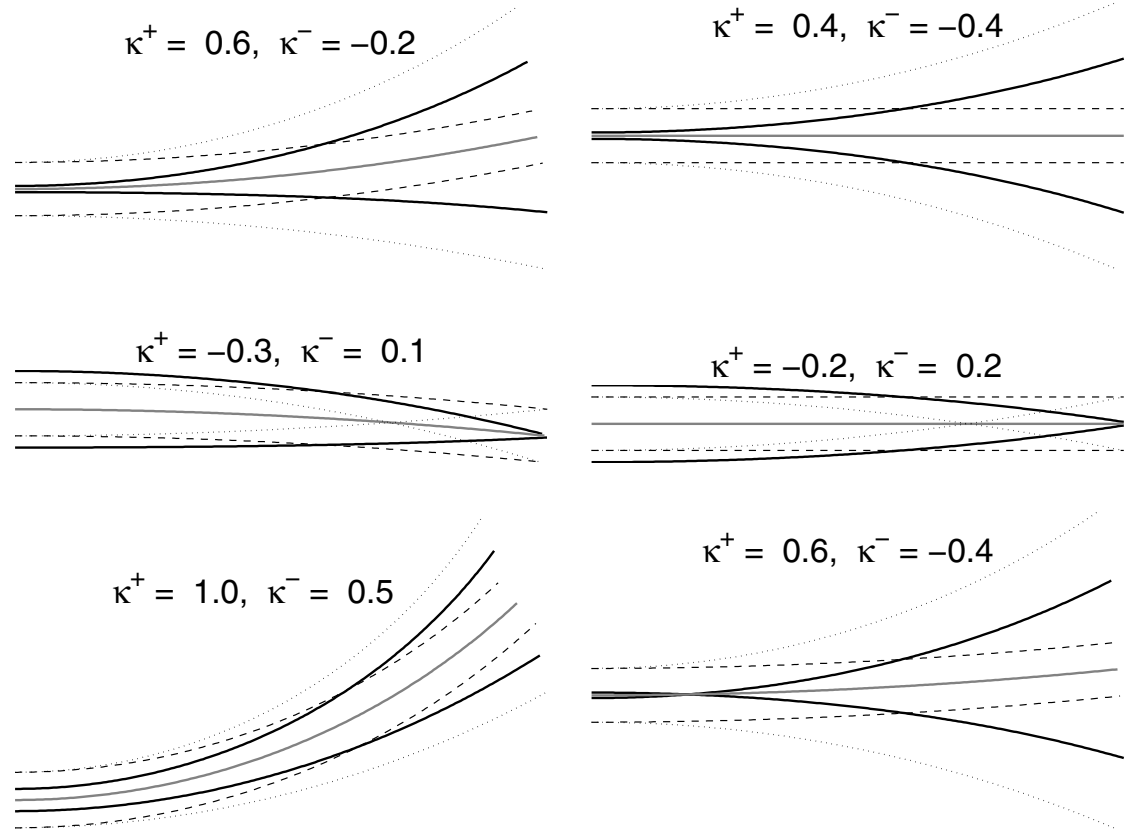

Figure 2. Stress-free configuration of birods. Gray lines represent the birods. Solid black lines represent the strands. Dotted lines show the shape of the strands before gluing them. The dashed lines are $d$-equidistant from the gray lines. When the dashed and solid line intersect, the transverse component of the gluing force changes sign.

i.e., when $\kappa^{+}=\kappa^{-}=0$, so we conclude that the reference state of the birod has a non-trivial structure.

A shooting method can be used to numerically solve the two-point boundary value problem (7.3) for general $\kappa^{+}$and $\kappa^{-}$. In Fig. 2 we present the stress-free configurations of birods made by gluing arcs of circles with the indicated intrinsic curvatures for parameter values $g=1, h=1, d=0.05$. The case $\kappa^{+}=0.6$ and $\kappa^{-}=-0.4$ exhibits an unphysical interpenetration of the strands. Such interpenetration could be avoided by using a more complicated constitutive equation for the microstress than (6.12a). Fig. 3 gives plots of the components in the moving frame of the microforce $\boldsymbol{n}^{\mathrm{c}}$ as functions of $s$. As remarked previously, when the strands have intrinsic curvatures of equal absolute value, but different sign, the reference configuration of the birod is straight. In such cases, a solution of the two-point boundary-value problem (7.3) corresponds to the solution of the reduced two-point boundary-value problem (7.4a). In such cases, the numerical solutions of both problems (7.3) and (7.4a) using the shooting method agree. Note that, since $n_{1}^{\mathrm{c}}=v \cos \vartheta-\mu \sin \vartheta$ and $n_{3}^{\mathrm{c}}=v \sin \vartheta+\mu \cos \vartheta$, the boundary conditions $\mu(1)=0, \nu(0)=\nu(1)=0$ and $\vartheta(0)=0$ yield $n_{1}^{\mathrm{c}}(0)=n_{1}^{\mathrm{c}}(1)=0$ and $n_{3}^{\mathrm{c}}(1)=0$.

If the strands are glued with the same concavities they tend to approach each other at $s=0$, and to separate at $s=1$. On the other hand, if the strands are glued with opposite concavities they tend to separate at $s=0$ and to approach at $s=1$. In both cases, at $s=s_{0} \in(0,1)$ the transverse component $\hat{f}_{3}=\hat{\boldsymbol{f}} \cdot \hat{\boldsymbol{d}}_{3}$ of the 

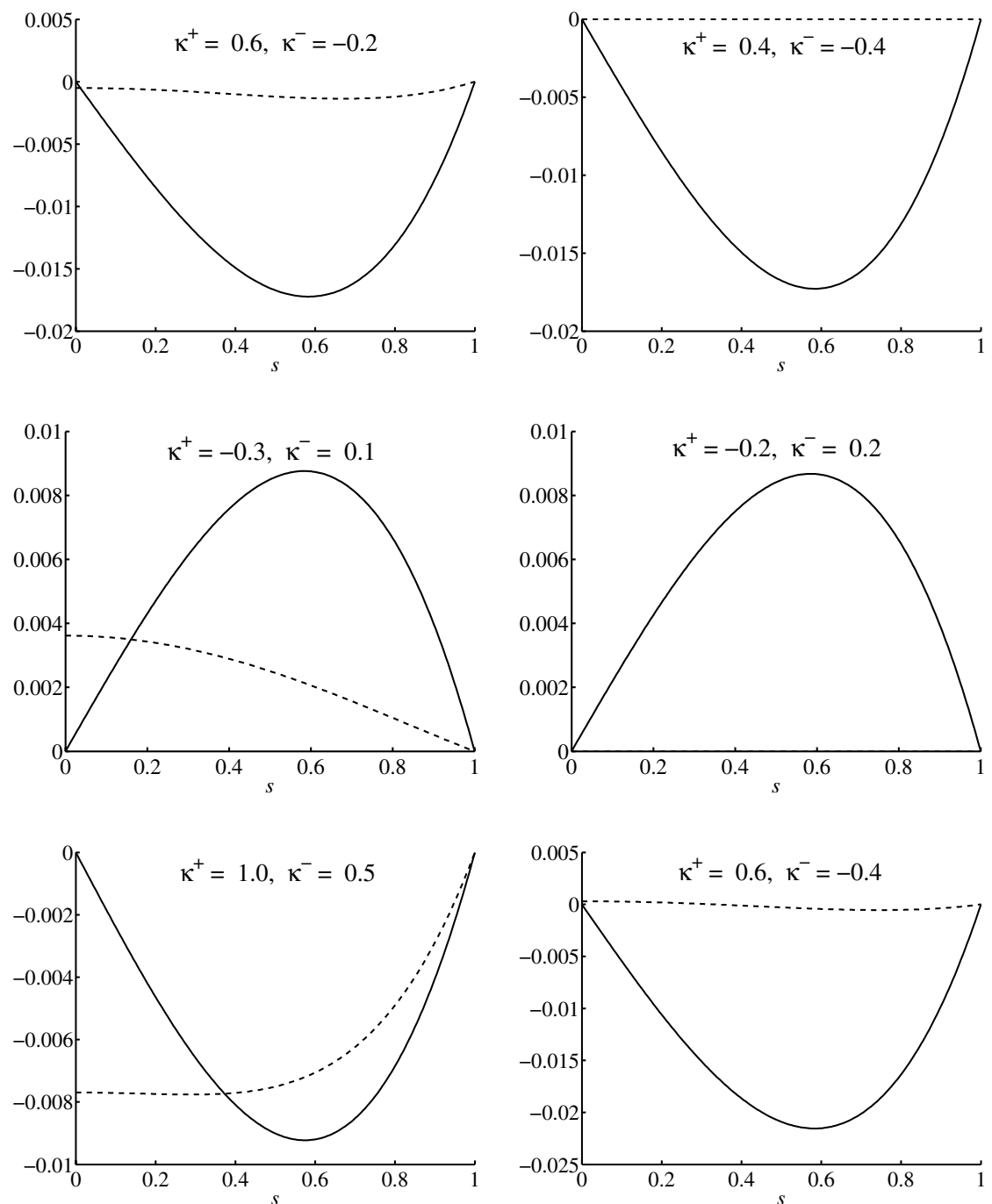

Figure 3. Components, in the moving frame, of the microforce $\hat{\boldsymbol{n}}^{\mathrm{c}}$ for the planar birod composed of two strands with the indicated intrinsic planar curvatures $\kappa^{ \pm}$. Solid lines represent $\hat{n}_{1}^{\mathrm{c}}=\hat{\boldsymbol{n}}^{\mathrm{c}} \cdot \hat{\boldsymbol{d}}_{1}$ while dashed lines indicate $\hat{n}_{3}^{\mathrm{c}}=\hat{\boldsymbol{n}}^{\mathrm{c}} \cdot \hat{\boldsymbol{d}}_{3}$ (which vanish identically in the case $\left.\kappa^{+}=-\kappa^{-}\right)$.

force $\hat{f}$ changes sign. In Fig. 4 we graph the two non-vanishing components in the moving frame of the gluing force $\hat{f}=\hat{\boldsymbol{n}}_{s}^{\mathrm{c}}$.

\section{Conclusions}

We have developed a new continuum mechanics theory of birods to describe two, tightly bound, elastic rods interacting elastically. The configuration of a birod 

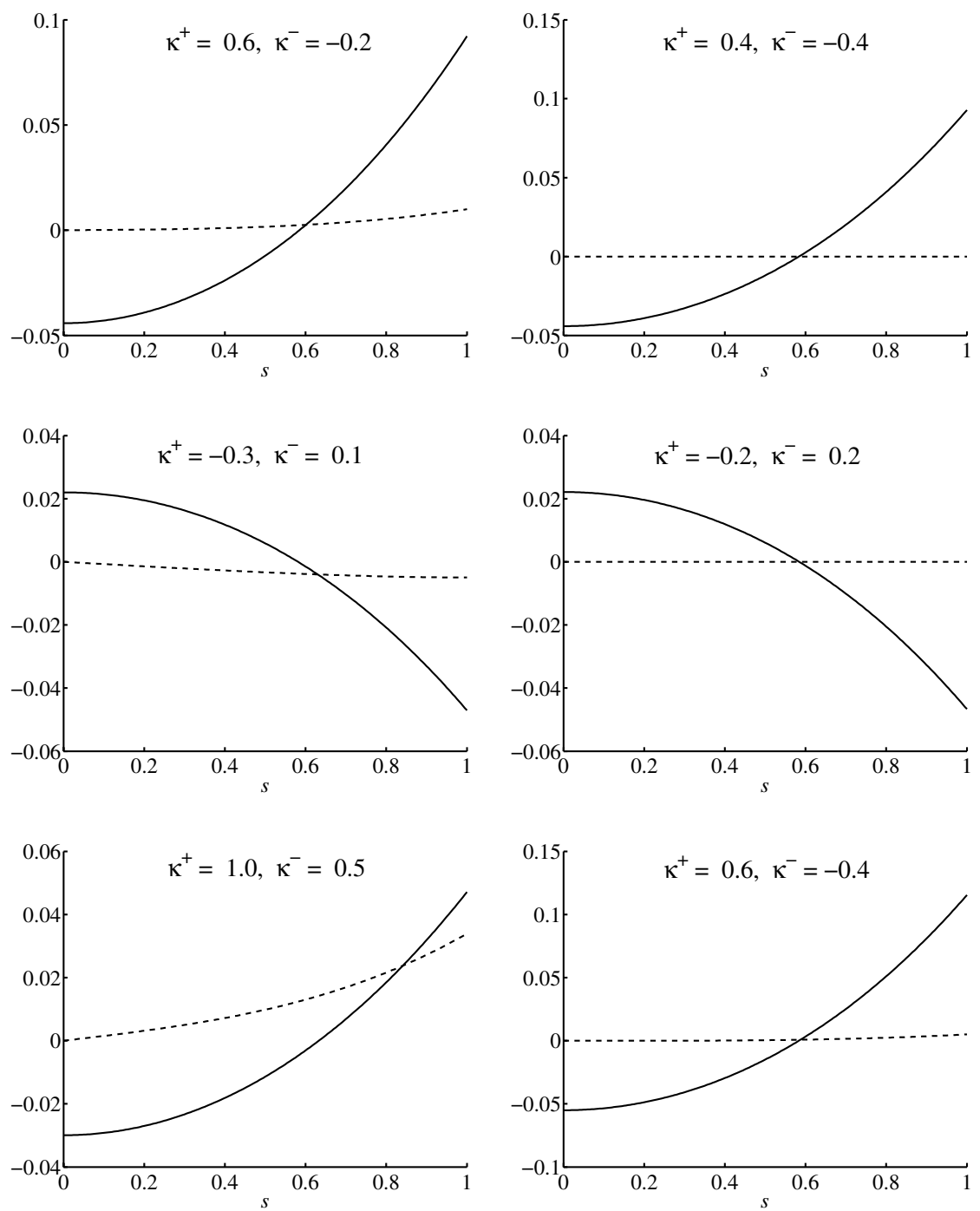

Figure 4. Components, in the moving frame, of the gluing force $\hat{f}$ for the planar birod composed of two strands with the indicated intrinsic curvatures $\kappa^{+}$and $\kappa^{-}$. Solid lines are for $\hat{f}_{1}=\hat{\boldsymbol{f}} \cdot \hat{\boldsymbol{d}}_{1}$, and dashed lines are for $\hat{f}_{3}=\hat{\boldsymbol{f}} \cdot \hat{\boldsymbol{d}}_{3}$.

is given by average macroscopic variables $(\boldsymbol{r}, \boldsymbol{R})$ analogous to a classic Cosserat rod, with the addition of internal variables $(\boldsymbol{w}, \boldsymbol{P})$ to describe the perturbation of each strand from the average. The dynamic balance laws $(3.11 \mathrm{a}, \mathrm{b})$ for the macroscopic variables $(\boldsymbol{r}, \boldsymbol{R})$ correspond exactly to the form of those for a classic Cosserat rod, although in general, and as shown explicitly for the linear case developed in Section 6, the constitutive relations involve both macroscopic and microscopic variables. The balance laws (3.11) for the microscopic variables take the form of a 
Cosserat rod subject to distributed forces and couples, with the simplification that the center-line variable is the macroscopic one.

We derive the balance laws in two ways. First, in Section 3, we proceed directly from the balance laws for the individual strands, and second, in Section 5.3 we start from Hamilton's principle with a rather general Lagrangian. Once an appropriate frame indifference is assumed, and some further natural restrictions on the assumed form of the kinetic energy terms in the Lagrangian are made, the Euler-Lagrange equations of the action principle reduce to the previously derived balance laws. The action principle approach is of interest because it clarifies the appropriate sense in which a birod is hyper-elastic (which is not entirely straightforward because of the presence of strain-gradient like terms), it provides the appropriate natural boundary conditions (which for the microscopic variables are not entirely obvious), and it opens the possibility of a variational analysis of stability properties of special solutions.

With regards to future work and applications of birods, the balance laws we derive are dynamic and, by choice of the constitutive relations, conservative. Thus for some simple classes of constitutive relations there is the immediate possibility of studying symmetry related, special solutions such as traveling waves. The static birod equations are simply obtained by suppressing time-dependent terms, and several interesting and non-trivial buckling problems immediately present themselves. Indeed, as shown in Section 7, even for the simplest linear constitutive relations, it is already non-trivial to compute the unstressed configuration of the birod structure, and resort must already be made to numerics. As already described in the Introduction, our primary motivation for developing the theory of birods is as a continuum approximation to rigid base models of DNA with sequence-dependent constitutive relations. In that context it seems inevitable that numerics will be the only way forward. Accordingly, the first challenge is to obtain sufficiently realistic, sequence-dependent constitutive relations for a birod. With the theory presented here it is possible to envision an approach to deriving appropriate constitutive relations for a continuum birod description of a rigid base model of DNA from molecular dynamics simulation in an analogous fashion to that proposed in [15] for obtaining continuum Cosserat rod parameters as an approximation to rigid base-pair models.

\section{Appendices}

\section{A. Properties of the Gibbs rotation vector}

Let $\boldsymbol{\eta}$ be the Gibbs rotation vector associated with the rotation tensor $\boldsymbol{P}$. Then

$$
\boldsymbol{P}=\boldsymbol{I}+\alpha\left[-(\boldsymbol{\eta} \cdot \boldsymbol{\eta}) \boldsymbol{I}+\boldsymbol{\eta}^{\times}+\boldsymbol{\eta} \otimes \boldsymbol{\eta}\right]
$$

where $\alpha=2 /(1+\boldsymbol{\eta} \cdot \boldsymbol{\eta})$. From the identity $\left(\boldsymbol{\eta}^{\times}\right)^{2}=\boldsymbol{\eta} \otimes \boldsymbol{\eta}-(\boldsymbol{\eta} \cdot \boldsymbol{\eta}) \boldsymbol{I}$ it follows that 


$$
\boldsymbol{P}=\boldsymbol{I}+\alpha \boldsymbol{\eta}^{\times}\left[\boldsymbol{I}+\boldsymbol{\eta}^{\times}\right]=\boldsymbol{I}+\alpha\left[\boldsymbol{I}+\boldsymbol{\eta}^{\times}\right] \boldsymbol{\eta}^{\times} .
$$

These expressions for $\boldsymbol{P}$ are helpful in proving the various identities given in the following lemmas, which are in turn useful in the development of our theory.

Lemma 1. Let $\boldsymbol{P}, \boldsymbol{\eta}$ and $\alpha$ be as above, then for all vectors $\boldsymbol{a}$ and $\boldsymbol{b}$ the following equations hold

i) $\boldsymbol{I}+\eta^{\times}=\boldsymbol{P}\left(\boldsymbol{I}-\eta^{\times}\right)=\left(\boldsymbol{I}-\eta^{\times}\right) \boldsymbol{P}$.

ii) $\boldsymbol{I}-\boldsymbol{\eta}^{\times}=\boldsymbol{P}^{T}\left(\boldsymbol{I}+\boldsymbol{\eta}^{\times}\right)=\left(\boldsymbol{I}+\boldsymbol{\eta}^{\times}\right) \boldsymbol{P}^{T}$.

iii) $\boldsymbol{a} \times \boldsymbol{P}^{T} \boldsymbol{b}+\alpha\left[\left(\boldsymbol{I}-\boldsymbol{\eta}^{\times}\right) \boldsymbol{a} \cdot \boldsymbol{P}^{T} \boldsymbol{b}\right] \boldsymbol{\eta}=\boldsymbol{a} \times \boldsymbol{b}+\alpha(\boldsymbol{\eta} \cdot \boldsymbol{a})\left(\boldsymbol{I}-\boldsymbol{\eta}^{\times}\right) \boldsymbol{b}$.

iv) $\boldsymbol{a} \times \boldsymbol{P} \boldsymbol{b}-\alpha\left[\left(\boldsymbol{I}+\boldsymbol{\eta}^{\times}\right) \boldsymbol{a} \cdot \boldsymbol{P} \boldsymbol{b}\right] \boldsymbol{\eta}=\boldsymbol{a} \times \boldsymbol{b}-\alpha(\boldsymbol{\eta} \cdot \boldsymbol{a})\left(\boldsymbol{I}+\boldsymbol{\eta}^{\times}\right) \boldsymbol{b}$.

The proofs involve straightforward algebra and the use of (A.1).

Lemma 2. Let $\boldsymbol{P}, \boldsymbol{\eta}$ and $\alpha$ be as above, and let $\boldsymbol{a}$ be a vector which is independent of $\boldsymbol{\eta}$. Then for any vector $\boldsymbol{b}$,
i) $\left[\frac{\partial(\boldsymbol{P} \boldsymbol{a})}{\partial \boldsymbol{\eta}}\right]^{T} \boldsymbol{b}=\alpha\left(\boldsymbol{I}+\eta^{\times}\right)\left(\boldsymbol{a} \times \boldsymbol{P}^{T} \boldsymbol{b}\right)$.
ii) $\left[\frac{\partial\left(\boldsymbol{P}^{T} \boldsymbol{a}\right)}{\partial \boldsymbol{\eta}}\right]^{T} \boldsymbol{b}=-\alpha\left(\boldsymbol{I}-\boldsymbol{\eta}^{\times}\right)(\boldsymbol{a} \times \boldsymbol{P} \boldsymbol{b})$.

Again, the proof of Lemma 2 is based on some algebra and Lemma 1.

\section{B. Partial derivatives of the strain energy density}

The use of Lemma 2, the definition (5.25) and the relations $\left(5.21^{ \pm}\right)$and $\left(5.22^{ \pm}\right)$ yields the following expressions for the partial derivatives of the strain energy density $\mathcal{W}$ with respect to its arguments:

$$
\begin{aligned}
& \frac{\partial \mathcal{W}}{\partial \mathbf{v}}=\left(\frac{\partial \mathbf{v}^{+}}{\partial \mathbf{v}}\right)^{T} \frac{\partial \tilde{\mathcal{W}}}{\partial \mathbf{v}^{+}}+\left(\frac{\partial \mathbf{v}^{-}}{\partial \mathbf{v}}\right)^{T} \frac{\partial \tilde{\mathcal{W}}}{\partial \mathbf{v}^{-}}=\boldsymbol{R}^{T}\left(\boldsymbol{n}^{+}+\boldsymbol{n}^{-}\right) \\
& \frac{\partial \mathcal{W}}{\partial \mathbf{u}}=\left(\frac{\partial \mathbf{u}^{+}}{\partial \mathbf{u}}\right)^{T} \frac{\partial \tilde{\mathcal{W}}}{\partial \mathbf{u}^{+}}+\left(\frac{\partial \mathbf{u}^{-}}{\partial \mathbf{u}}\right)^{T} \frac{\partial \tilde{\mathcal{W}}}{\partial \mathbf{u}^{-}}+\left(\frac{\partial \mathbf{v}^{+}}{\partial \mathbf{u}}\right)^{T} \frac{\partial \tilde{\mathcal{W}}}{\partial \mathbf{v}^{+}}+\left(\frac{\partial \mathbf{v}^{-}}{\partial \mathbf{u}}\right)^{T} \frac{\partial \tilde{\mathcal{W}}}{\partial \mathbf{v}^{-}} \\
& =\boldsymbol{R}^{T}\left[\boldsymbol{m}^{+}+\boldsymbol{m}^{-}+\boldsymbol{w} \times\left(\boldsymbol{n}^{+}-\boldsymbol{n}^{-}\right)\right], \\
& \frac{\partial \mathcal{W}}{\partial \mathbf{v}^{\mathrm{c}}}=\left(\frac{\partial \mathbf{v}^{+}}{\partial \mathbf{v}^{\mathrm{c}}}\right)^{T} \frac{\partial \tilde{\mathcal{W}}}{\partial \mathbf{v}^{+}}+\left(\frac{\partial \mathbf{v}^{-}}{\partial \mathbf{v}^{\mathrm{c}}}\right)^{T} \frac{\partial \tilde{\mathcal{W}}}{\partial \mathbf{v}^{-}}=\boldsymbol{R}^{T}\left(\boldsymbol{n}^{+}-\boldsymbol{n}^{-}\right), \\
& \frac{\partial \mathcal{W}}{\partial \mathbf{u}^{\mathrm{c}}}=\left(\frac{\partial \mathbf{u}^{+}}{\partial \mathbf{u}^{\mathrm{c}}}\right)^{T} \frac{\partial \tilde{\mathcal{W}}}{\partial \mathbf{u}^{+}}+\left(\frac{\partial \mathbf{u}^{-}}{\partial \mathbf{u}^{\mathrm{c}}}\right)^{T} \frac{\partial \tilde{\mathcal{W}}}{\partial \mathbf{u}^{-}} \\
& =\boldsymbol{R}^{T}\left[\boldsymbol{m}^{+}-\boldsymbol{m}^{-}-\boldsymbol{\eta} \times\left(\boldsymbol{m}^{+}+\boldsymbol{m}^{-}\right)\right], \\
& \frac{\partial \mathcal{W}}{\partial \mathbf{w}}=\frac{\partial \tilde{\mathcal{W}}}{\partial \mathbf{w}}+\left(\frac{\partial \mathbf{v}^{+}}{\partial \mathbf{w}}\right)^{T} \frac{\partial \tilde{\mathcal{W}}}{\partial \mathbf{v}^{+}}+\left(\frac{\partial \mathbf{v}^{-}}{\partial \mathbf{w}}\right)^{T} \frac{\partial \tilde{\mathcal{W}}}{\partial \mathbf{v}^{-}} \\
& =\frac{\partial \widetilde{\mathcal{W}}}{\partial \mathbf{w}}-\boldsymbol{R}^{T}\left[\boldsymbol{u} \times\left(\boldsymbol{n}^{+}-\boldsymbol{n}^{-}\right)\right],
\end{aligned}
$$




$$
\begin{gathered}
\frac{\partial \mathcal{W}}{\partial \boldsymbol{\eta}}=\frac{\partial \tilde{\mathcal{W}}}{\partial \boldsymbol{\eta}}+\left(\frac{\partial \mathbf{v}^{+}}{\partial \boldsymbol{\eta}}\right)^{T} \frac{\partial \widetilde{\mathcal{W}}}{\partial \mathbf{v}^{+}}+\left(\frac{\partial \mathbf{v}^{-}}{\partial \boldsymbol{\eta}}\right)^{T} \frac{\partial \tilde{\mathcal{W}}}{\partial \mathbf{v}^{-}}+\left(\frac{\partial \mathbf{u}^{+}}{\partial \boldsymbol{\eta}}\right)^{T} \frac{\partial \tilde{\mathcal{W}}}{\partial \mathbf{u}^{+}}+\left(\frac{\partial \mathbf{u}^{-}}{\partial \boldsymbol{\eta}}\right)^{T} \frac{\partial \tilde{\mathcal{W}}}{\partial \mathbf{u}^{-}} \\
=\frac{\partial \mathcal{W}}{\partial \boldsymbol{\eta}}-\alpha \boldsymbol{R}^{T}\left\{\boldsymbol{v} \times\left(\boldsymbol{n}^{+}-\boldsymbol{n}^{-}\right)+\boldsymbol{w}_{s} \times\left(\boldsymbol{n}^{+}+\boldsymbol{n}^{-}\right)+\boldsymbol{u} \times\left(\boldsymbol{m}^{+}-\boldsymbol{m}^{-}\right)\right. \\
-\boldsymbol{\eta} \times\left[\boldsymbol{v} \times\left(\boldsymbol{n}^{+}+\boldsymbol{n}^{-}\right)+\boldsymbol{w}_{s} \times\left(\boldsymbol{n}^{+}-\boldsymbol{n}^{-}\right)+\boldsymbol{u} \times\left(\boldsymbol{m}^{+}+\boldsymbol{m}^{-}\right)\right] \\
\left.+\boldsymbol{\eta}^{\nabla} \times\left(\boldsymbol{P}^{T} \boldsymbol{m}^{+}+\boldsymbol{P} \boldsymbol{m}^{-}\right)\right\} .
\end{gathered}
$$

Expressions for the partial derivatives of the kinetic energy density $\mathcal{K}$ with respect to its arguments are obtained from the above with the help of the variable interchange (5.31).

Acknowledgements. It is a pleasure to be able to thank K.R. RAJAGOPAL, C.J. BENHAM, and members of the LCVM ${ }^{2}$ group at the EPFL for helpful discussions concerning this research. The work was supported in part by the Swiss National Science Foundation.

\section{References}

1. Angeles, J.: Rational Kinematics. Springer-Verlag, New York, 1988

2. Antman, S.S.: Nonlinear Problems of Elasticity. 2nd ed. Springer-Verlag, New York, 2004

3. ARnOld, V.I., Kozlov, V.V., NEǏshTADT, A.I.: Mathematical Aspects of Classical and Celestial Mechanics, 2nd ed. Springer-Verlag, Berlin, 1997

4. Barbi, M., Cocco, S., PeYrard, M., RufFo, S.: A twist opening model for DNA. J. Biol. Phys. 24, 358-369 (1999)

5. BAUER, W.R. CRICK, F.H.C., WHITE, J.H.: Superhelical DNA. Scientific American 243, 100-113 (1980)

6. Benham, C.J.: Elastic model of supercoiling. Proc. Natl. Acad. Sci. 74, 2397-2401 (1977)

7. BENHAM, C.J.: Theoretical analysis of heteropolymeric transitions in superhelical DNA molecules of specified sequence. J. Chem. Phys. 92, 6294-6305 (1990)

8. BENHAM, C.J.: Energetics of the strand separation transition in superhelical DNA. J. Mol. Biol. 225, 835-847 (1992)

9. CAPRIZ, G.: Continua with Microstructure. Springer-Verlag, New York, 1989

10. Coleman, B.D., SwigOn, D.: Theory of supercoiled elastic rings with self-contact and its application to DNA plasmids. J. Elasticity 60, 173-221 (2000)

11. Cosserat, E., Cosserat, F.: Théorie des Corps Déformables. Hermann \& Fils, Paris, 1909

12. Costello, G.A.: Theory of Wire Rope. Springer-Verlag, New York, 1990

13. Fraser, W.B., STUMP, D.M.: The equilibrium of the convergence point in two-strand yarn plying. Int. J. of Solids and Structures 35, 285-298 (1998)

14. Gaeta, G., Reiss, C., Peyrard, M., Dauxois, T.: Simple models of nonlinear DNA dynamics. Rivista del Nuovo Cimento 17, 1-48 (1994)

15. GONZALEZ, O., MADDOCKS, J.H.: Extracting parameters for base-pair level models of DNA from molecular dynamics simulations. Theoretical Chemistry Accounts 106, 76$82(2001)$

16. LEBRET, M.: Twist and writhing in short circular DNA according to first-order elasticity. Biopolymers 23, 1835-1867 (1984)

17. MANNING, R.S., MADDOCKS, J.H., KAHN, J.D.: A continuum rod model of sequencedependent DNA structure. J. Chem. Phys. 105 , 5626-5646 (1996) 
18. MARSDEN, J.E., RATIU, T.S.: Introduction to Mechanics and Symmetry. Springer-Verlag, New York, 1999

19. MOAKHER, M.: Means and averaging in the group of rotations. SIAM J. Matrix Anal. Appl. 24, 1-16 (2002)

20. PEYRARD, M., BishoP, A.R.: Statistical mechanics of a nonlinear model for DNA denaturation. Phys. Rev. Lett. 62, 2755-2758 (1989)

21. Prohofsky, E.: Statistical Mechanics and Stability of Macromolecules: Application to Bond Disruption, Base Pair Separation, Melting, and Drug Dissociation of the DNA Double Helix. Cambridge University Press, Cambridge, 1995

22. Rajagopal, K.R., TAO, L.: Mechanics of Mixtures. World Scientific, Singapore, 1995

23. RizZi, M.A., WhitMAn, A.B., DeSiLVA, C.N.: A mathematical model of the spine based on mixture theory of directed curves. Acta Mechanica 21, 241-260 (1975)

24. SCHLICK, T.: Modeling superhelical DNA: recent analytical and dynamical approaches. Current Opinion in Structural Biology 5, 245-262 (1995)

25. Swigon, D., Coleman, B.D., ToBIAS, I.: The elastic rod model for DNA and its application to the tertiary structure of DNA minicircles in mononucleosomes. Biophysical Journal 74, 2515-2530 (1998)

26. TADJBAKHSH, I.: The variational theory of the plane motion of the extensible elastica. Int. J. Eng. Sci. 4, 33-450 (1966)

27. Thompson, J.M.T., van DER HeIJden, G.H.M., NeukIRCH, S.: Supercoiling of DNA plasmids: mechanics of the generalized ply. Proc. R. Soc. Lond. A 458, 959-985 (2000)

28. TOBIAS, I., OLSON, W.K.: The effect of intrinsic curvature on supercoiling: predictions of elasticity theory. Biopolymers 33, 639-646 (1993)

29. Toupin, R.A.: Theories of elasticity with couple-stress. Arch. Ration. Mech. Anal. 17, 85-112 (1964)

30. TRuesdell, C.: Six Lectures on Modern Natural Philosophy. Springer-Verlag, New York, 1966

31. Truesdell, C., Noll, W.: The Non-Linear Field Theories of Mechanics, S. S. Antman, Ed., 3rd ed. Springer, New York, 2004

32. Tsiotras, P., Junkins, J., Schaub, H.: Higher order Cayley-transforms with applications to attitude representations. Journal of Guidance, Control, and Dynamics 20, 528-534 (1997)

33. YAKUSHEVICH, L.V.: Nonlinear Physics of DNA. John Wiley \& Sons, Chichester, 1998

34. YAKUSHEVICH, L.V.: Is DNA a nonlinear system where solitary conformation waves are possible? J. Biosci. 26, 305-313 (2001)

35. Yomosa, S.: Soliton excitations in deoxyribonucleic acid (DNA) double helices. Phys. Rev. A. 27, 2120-2125 (1983)

Laboratoire de Modélisation Mathématique et Numérique dans les Sciences de l'Ingénieur

Ecole Nationale d'Ingénieurs de Tunis

B.P. 37, 1002 Tunis, Tunisia e-mail: Maher.Moakher@enit.rnu.tn

and

Institut Mathématique B

Ecole Polytechnique Fédérale de Lausanne

CH-1015 Lausanne, Switzerland

e-mail: John.Maddocks@epfl.ch 